\title{
Meson-meson scattering within one-loop chiral perturbation theory and its unitarization
}

\author{
A. Gómez Nicola and J. R. Peláez \\ Departamento de Física Teórica II, Universidad Complutense de Madrid, 28040-Madrid, Spain
}

(Received 7 September 2001; published 30 January 2002)

\begin{abstract}
We present a complete one-loop calculation of all the two-meson scattering amplitudes within the framework of SU(3) chiral perturbation theory, which includes pions, kaons, and the eta. In addition, we have unitarized these amplitudes with the coupled channel inverse amplitude method, which simultaneously ensures the good low energy properties of chiral perturbation theory and unitarity. We show how this method provides a remarkable description of meson-meson scattering data up to $1.2 \mathrm{GeV}$ including the scattering lengths and the generation of seven light resonances, which is consistent with previous determinations of the chiral parameters. Particular attention is paid to discussing the differences and similarities of this work with previous analyses in the literature.
\end{abstract}

DOI: 10.1103/PhysRevD.65.054009

PACS number(s): 13.75.Lb, 11.80.Et, 12.39.Fe, 14.40. $-\mathrm{n}$

\section{INTRODUCTION}

In the last 20 years, chiral perturbation theory (ChPT) [1-3] has emerged as a powerful tool to describe the interactions of the lightest mesons. These particles are considerably lighter than the rest of the hadrons, which is nowadays understood as a consequence of the spontaneous breaking of the $\mathrm{SU}(3)_{L} \times \mathrm{SU}(3)_{R}$ chiral symmetry down to $\mathrm{SU}(3)_{L+R}$, which would be present in QCD if the three lightest quarks were massless. In such a case, the light mesons would correspond to the massless Goldstone bosons associated with spontaneous chiral symmetry breaking. Of course, quarks are not massless, but their masses are so small compared to the typical hadronic scales $O(1 \mathrm{GeV})$ that their explicit symmetry breaking effect also translates into a small mass for the lightest mesons, which become pseudo Goldstone bosons. Hence, the three pions correspond to the pseudo Goldstone bosons of the SU(2) spontaneous breaking that would occur if only the $u$ and $d$ quarks were massless, which is a remarkably good approximation. Similarly, the meson octet formed by the pions, the kaons, and the eta can be identified with the eight pseudo Goldstone bosons associated with the SU(3) breaking when the $s$ quark is also included.

The low energy interactions of pions, kaons, and the eta can be described in terms of an effective Lagrangian that follows the $\mathrm{SU}(3)_{L} \times \mathrm{SU}(3)_{R} \rightarrow \mathrm{SU}(3)_{L+R}$ spontaneous symmetry breaking pattern. If we do not include any additional field apart from the pseudo Goldstone bosons, this description will be valid only for energies much below the scale where new states appear. That is, the effective ChPT Lagrangian provides just a low energy description. As a consequence we can organize all the possible terms that respect the symmetry requirements in a derivative (and mass) expansion. Therefore, any amplitude is obtained as a perturbative expansion in powers of the external momenta and the quark masses. The importance of this formalism is that the theory is renormalizable and predictive, in the following sense: all loop divergences appearing at a given order in the expansion can be absorbed by a finite number of counterterms, or low energy constants, that appear in the Lagrangian at that very same order. Thus, order by order, the theory is finite and depends on a few parameters that can be determined experi- mentally. Once these parameters are known, any other calculation at that order becomes a prediction. Basically, these are the main ideas underlying ChPT, which has proved very successful in describing low energy hadron phenomenology (for reviews see [4]).

Despite the success of this approach, it is unfortunately limited to low energies (usually, less than $500 \mathrm{MeV}$ ). That is the reason why, over the last few years, there has been a growing interest in extending the applicability range of the chiral expansion to higher energies. Of course, this requires the use of nonperturbative methods to improve the high energy behavior of ChPT amplitudes. These methods include the explicit introduction of heavier resonant states in the Lagrangian [5], resummation of diagrams in a LippmannSchwinger or Bethe-Salpeter approach [6], or other methods that unitarize the amplitudes like the inverse amplitude method (IAM) [7,8]. The last method has been generalized to allow for a coupled channel formalism [9], yielding a successful description of the meson-meson scattering amplitudes up to $1.2 \mathrm{GeV}$, and even generating dynamically seven light resonances.

In principle, these methods recover at low energies the good properties of ChPT, since they use part of the perturbative information. However, it should be noted that, so far, the full results to one loop for all the meson-meson scattering processes are not available in the literature. At present, only the $\pi \pi \rightarrow \pi \pi$ [10], $K \pi \rightarrow K \pi$ [10], $\eta \pi \rightarrow \eta \pi$ [10], and the two independent $K^{+} K^{-} \rightarrow K^{+} K^{-}, K^{+} K^{-} \rightarrow K^{0} \bar{K}^{0}$ [11] amplitudes have been obtained in the SU(3) ChPT framework, although with different procedures and notation. As a consequence, the IAM has been applied rigorously only to the $\pi \pi$, $K \bar{K}$ final states, whereas for a complete treatment of the whole low energy meson-meson scattering, additional approximations had to be made [9]. In particular, the lowest order expansion could not be recovered complete up to $O\left(p^{4}\right)$, thus spoiling the scattering lengths and, in addition, it was not possible to compare directly with the low energy parameters of standard ChPT in dimensional regularization and the modified minimal subtraction $\overline{\mathrm{MS}}-1$ scheme.

In this work, we have calculated all the meson-meson scattering amplitudes at one loop in ChPT. There are three 
amplitudes that have never appeared published in the literature: $K \eta \rightarrow K \eta, \eta \eta \rightarrow \eta \eta$, and $K \pi \rightarrow K \eta$. The other five have been recalculated independently and all of them are given together in a unified notation, ensuring exact perturbative unitarity and also correcting previous misprints. Then, we have applied the coupled channel IAM to describe the whole meson-meson scattering below $1.2 \mathrm{GeV}$, including low energy data like scattering lengths. This new calculation allows for a direct comparison with the standard low energy constants of ChPT and that is why we have made a considerable effort to estimate the uncertainties in all our results, which are in very good agreement with the present determinations obtained from low energy data without unitarization. The main differences of this work from [9] are that we consider the full one-loop results for the amplitudes, ensuring their finiteness and scale independence in dimensional regularization, we take into account the new processes mentioned above, and we are able to describe the low energy region more accurately. This had already been achieved for the $\pi \pi$, $K \bar{K}$ system only in [11], but here we complete this task for all meson-meson scattering.

The paper is organized as follows. In Sec. II we review the main features of the meson-meson scattering calculations at one loop in ChPT. The final results for the amplitudes are collected in Appendix B because of their length. The definition of partial waves and unitarity is discussed in Sec. III, and the IAM is presented in Sec. IV. In Sec. V, we review the available data on meson-meson scattering. In Secs. VI and VII we first use the IAM with present determinations of the low energy constants and next make a fit to the data reviewed in Sec. V. Our conclusions are summarized in Sec. VIII. Apart from the amplitudes in Appendix B, we have also collected some useful formulas in Appendix A.

\section{MESON-MESON SCATTERING AT ONE LOOP}

The lowest order Lagrangian for SU(3) chiral perturbation theory is

$$
\mathcal{L}_{2}=\frac{f_{0}^{2}}{4}\left\langle\partial_{\mu} U^{\dagger} \partial^{\mu} U+M_{0}\left(U+U^{\dagger}\right)\right\rangle
$$

where $f_{0}$ is the pion decay constant in the $\mathrm{SU}(3)$ chiral limit and the angular brackets stand for the trace of the $3 \times 3$ matrices. The matrix $U$ collects the pseudo Goldstone boson fields $\pi, K, \eta$ through $U(\Phi)=\exp \left(i \sqrt{2} \Phi / f_{0}\right)$, where

$$
\Phi(x)=\left(\begin{array}{ccc}
\frac{1}{\sqrt{2}} \pi^{0}+\frac{1}{\sqrt{6}} \eta & \pi^{+} & K^{+} \\
\pi^{-} & -\frac{1}{\sqrt{2}} \pi^{0}+\frac{1}{\sqrt{6}} \eta & K^{0} \\
K^{-} & \bar{K}^{0} & -\frac{2}{\sqrt{6}} \eta
\end{array}\right)
$$

and $M_{0}$ is the tree level mass matrix. Throughout this paper we will be assuming the isospin limit, so that $M_{0}$ is given by
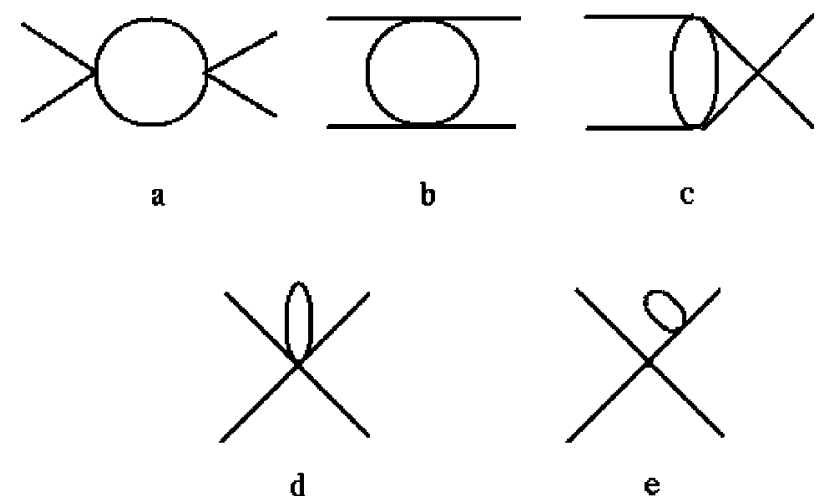

FIG. 1. Generic one-loop Feynman diagrams that have to be evaluated in meson-meson scattering.

$$
M_{0}=\left(\begin{array}{ccc}
M_{0 \pi}^{2} & 0 & 0 \\
0 & M_{0 \pi}^{2} & 0 \\
0 & 0 & 2 m_{0 K}^{2}-M_{0 \pi}^{2}
\end{array}\right) .
$$

As a matter of fact, from these definitions, it can be easily seen that the tree level masses satisfy the Gell-Mann-Okubo relation [12] $4 M_{0 K}^{2}-M_{0 \pi}^{2}-3 M_{0 \eta}^{2}=0$, which will be very useful for simplifying the amplitudes.

From the Lagrangian in Eq. (1), one can obtain the $O\left(p^{2}\right)$ amplitudes just by calculating the corresponding tree level Feynman diagrams. In order to obtain the $O\left(p^{4}\right)$ contributions, one has to consider loop diagrams, whose generic topology is given in Fig. 1, which will generate UV divergences. If loop integrals are regularized with dimensional regularization, which preserves the chiral symmetry constraints, the divergences can be reabsorbed in the chiral parameters $L_{i}$ of the fourth order Lagrangian:

$$
\begin{aligned}
\mathcal{L}_{4}= & L_{1}\left\langle\partial_{\mu} U^{\dagger} \partial^{\mu} U\right\rangle^{2}+L_{2}\left\langle\partial_{\mu} U^{\dagger} \partial_{\nu} U\right\rangle\left\langle\partial^{\mu} U^{\dagger} \partial^{\nu} U\right\rangle \\
& +L_{3}\left\langle\partial_{\mu} U^{\dagger} \partial^{\mu} U \partial_{\nu} U^{\dagger} \partial^{\nu} U\right\rangle+L_{4}\left\langle\partial_{\mu} U^{\dagger} \partial^{\mu} U\right\rangle\left\langle U^{\dagger} M_{0}\right. \\
& \left.+M_{0}^{\dagger} U\right\rangle+L_{5}\left\langle\partial_{\mu} U^{\dagger} \partial^{\mu} U\left(U^{\dagger} M_{0}+M_{0}^{\dagger} U\right)\right\rangle+L_{6}\left\langle U^{\dagger} M_{0}\right. \\
& \left.+M_{0}^{\dagger} U\right\rangle^{2}+L_{7}\left\langle U^{\dagger} M_{0}-M_{0}^{\dagger} U\right\rangle^{2}+L_{8}\left\langle M_{0}^{\dagger} U M_{0}^{\dagger} U\right. \\
& \left.+U^{\dagger} M_{0} U^{\dagger} M_{0}\right\rangle,
\end{aligned}
$$

where the terms that couple to external sources, like gauge fields, are omitted $[2,3]$. The $L_{i}$ constants are related to the renormalized $L_{i}^{r}(\mu)$ generically as $L_{i}=L_{i}^{r}(\mu)+\Gamma_{i} \lambda \quad$ [3] where $\mu$ is the $\overline{\mathrm{MS}}-1$ renormalization scale,

$$
\lambda=\frac{\mu^{d-4}}{16 \pi^{2}}\left[\frac{1}{d-4}-\frac{1}{2}(\log 4 \pi-\gamma+1)\right],
$$

$\gamma$ is the Euler constant, and the $\Gamma_{i}$ coefficients can be found in [3]. We remark that the $L_{3}$ and $L_{7}$ constants are not renormalized and are therefore scale independent, i.e., $\Gamma_{3}=\Gamma_{7}$ $=0$.

Thus, up to fourth order one has to consider the tree level diagrams from $O\left(p^{2}\right)$ and $O\left(p^{4}\right)$, together with the one-loop diagrams in Fig. 1. We stress that mass and wave function renormalizations should be accounted for to the same order. 
The latter are schematically represented by the tadpole diagram (e) in Fig. 1. As is customary, we define the bare fields in terms of the renormalized ones as $\pi=Z_{\pi}^{1 / 2} \pi^{\text {ren }}$ and so on for the kaons and eta, so that scalar fields have finite canonical kinetic terms. Taking into account all the different contributions from diagrams of type (e) in Fig. 1 plus those tree level diagrams coming from $\mathcal{L}_{4}$, one obtains

$$
\begin{aligned}
Z_{\pi}= & +\frac{4}{3} \mu_{\pi}+\frac{2}{3} \mu_{K}-\frac{4 \lambda}{3 f_{0}^{2}}\left(2 M_{0 \pi}^{2}+M_{0 K}^{2}\right) \\
& -\frac{8}{f_{0}^{2}}\left[2 L_{4}^{r} M_{0 K}^{2}+\left(L_{4}^{r}+L_{5}^{r}\right) M_{0 \pi}^{2}\right], \\
Z_{K}=1 & +\frac{1}{2} \mu_{\pi}+\mu_{K}+\frac{1}{2} \mu_{\eta}-\frac{2 \lambda}{3 f_{0}^{2}}\left(M_{0 \pi}^{2}+5 M_{0 K}^{2}\right) \\
- & \frac{8}{f_{0}^{2}}\left[\left(2 L_{4}^{r}+L_{5}^{r}\right) M_{0 K}^{2}+L_{4}^{r} M_{0 \pi}^{2}\right], \\
Z_{\eta}=1 & +2 \mu_{K}-\frac{4 \lambda}{f_{0}^{2}} M_{0 K}^{2}-\frac{8}{3 f_{0}^{2}}\left[\left(3 L_{4}^{r}-L_{5}^{r}\right) M_{0 \pi}^{2}\right. \\
& \left.+2\left(3 L_{4}^{r}+2 L_{5}^{r}\right) M_{0 K}^{2}\right],
\end{aligned}
$$

where

$$
\mu_{i}=\frac{M_{i}^{2}}{32 \pi^{2} f_{0}^{2}} \log \frac{M_{i}^{2}}{\mu^{2}}
$$

with $i=\pi, K, \eta$.

Note that the wave function renormalization constants $Z_{i}$ contain a divergent part and they are scale dependent. As for the mass renormalizations, the physical pion and kaon masses are given in terms of the tree level ones as [3]

$$
\begin{gathered}
M_{\pi}^{2}=M_{0 \pi}^{2}\left[1+\mu_{\pi}-\frac{\mu_{\eta}}{3}+\frac{16 M_{0 K}^{2}}{f_{0}^{2}}\left(2 L_{6}^{r}-L_{4}^{r}\right)\right. \\
\left.+\frac{8 M_{0 \pi}^{2}}{f_{0}^{2}}\left(2 L_{6}^{r}+2 L_{8}^{r}-L_{4}^{r}-L_{5}^{r}\right)\right], \\
M_{K}^{2}=M_{0 K}^{2}\left[1+\frac{2 \mu_{\eta}}{3}+\frac{8 M_{0 \pi}^{2}}{f_{0}^{2}}\left(2 L_{6}^{r}-L_{4}^{r}\right)\right. \\
\left.\quad+\frac{8 M_{0 K}^{2}}{f_{0}^{2}}\left(4 L_{6}^{r}+2 L_{8}^{r}-2 L_{4}^{r}-L_{5}^{r}\right)\right], \\
M_{\eta}^{2}=M_{0 \eta}^{2}\left[1+2 \mu_{K}-\frac{4}{3} \mu_{\eta}+\frac{8 M_{0 \eta}^{2}}{f_{0}^{2}}\left(2 L_{8}^{r}-L_{5}^{r}\right)\right. \\
\left.+\frac{8}{f_{0}^{2}}\left(2 M_{0 K}^{2}+M_{0 \pi}^{2}\right)\left(2 L_{6}^{r}-L_{4}^{r}\right)\right]+M_{0 \pi}^{2}\left[-\mu_{\pi}+\frac{2}{3} \mu_{K}\right. \\
\left.+\frac{1}{3} \mu_{\eta}\right]+\frac{128}{9 f_{0}^{2}}\left(M_{0 K}^{2}-M_{0 \pi}^{2}\right)^{2}\left(3 L_{7}+L_{8}^{r}\right) .
\end{gathered}
$$

According to the chiral power counting, we have to use Eqs. (6) and (8) only in the tree level part of the amplitudes. In fact, the mass renormalization Eq. (8) affects only the mass terms coming from the Lagrangian in Eq. (1) and not the masses coming from the kinematics of the corresponding process. As will be seen below, we will not need the mass renormalization of $M_{\eta}$ in any of our expressions.

The meson decay constants are also modified to one loop. It will be convenient for our purposes to write all the oneloop amplitudes in terms of a single decay constant, which we have chosen to be $f_{\pi}$. For that reason and for an easier comparison with previous results in the literature, we also give here the result for the meson decay constants to one loop [3]:

$$
\begin{gathered}
f_{\pi}=f_{0}\left[1-2 \mu_{\pi}-\mu_{K}+\frac{4 M_{0 \pi}^{2}}{f_{0}^{2}}\left(L_{4}^{r}+L_{5}^{r}\right)+\frac{8 M_{0 K}^{2}}{f_{0}^{2}} L_{4}^{r}\right], \\
f_{K}=f_{0}\left[1-\frac{3 \mu_{\pi}}{4}-\frac{3 \mu_{K}}{2}-\frac{3 \mu_{\eta}}{4}+\frac{4 M_{0 \pi}^{2}}{f_{0}^{2}} L_{4}^{r}\right. \\
\left.+\frac{4 M_{0 K}^{2}}{f_{0}^{2}}\left(2 L_{4}^{r}+L_{5}^{r}\right)\right], \\
f_{\eta}=f_{0}\left[1-3 \mu_{K}+\frac{4 L_{4}^{r}}{f_{0}^{2}}\left(M_{0 \pi}^{2}+2 M_{0 K}^{2}\right)+\frac{4 M_{0 \eta}^{2}}{f_{0}^{2}} L_{5}^{r}\right] .
\end{gathered}
$$

It is important to stress that both the physical masses in Eq. (8) and the decay constants in Eq. (9) are finite and scale independent.

Therefore, the one-loop ChPT scattering amplitude (renormalized and scale independent) for a given process will have the generic form

$$
T(s, t, u)=T_{2}(s, t, u)+T_{4}^{\mathrm{pol}}(s, t, u)+T_{4}^{\mathrm{uni}}(s, t, u)
$$

where $s, t, u$ are the Mandelstam variables. Here, $T_{2}$ is the tree level contribution from the Lagrangian in Eq. (1), whereas $T_{4}^{\text {pol }}$ contains the fourth order terms which are polynomials in $s, t, u$. Those polynomials have four possible origins: tree level terms from the Lagrangian in Eq. (4) proportional to $L_{i}^{r}$, other polynomial terms proportional to $L_{i}$ with $i$ $=4, \ldots, 8$ coming from the mass and decay constant renormalization in Eqs. (8) and (9), terms proportional to $\mu_{i}$ coming from tadpole diagrams [(d) and (e) in Fig. 1], and finally pure polynomial fourth order terms which stem from our parametrization of the one-loop functions (see Appendix A). Let us remark that, for technical reasons explained in Sec. III B, we have chosen to write all our amplitudes in terms of $f_{\pi}$ only since, using Eqs. (9), $f_{K}$ and $f_{\eta}$ can be expressed in terms of $f_{\pi}, L_{4}^{r}$, and $L_{5}^{r}$. In addition, $T_{4}^{\text {uni }}$ stands for the contribution of diagrams (a), (b), and (c) in Fig. 1. These contributions not only contain the imaginary parts required by unitarity but also yield the correct analytic structure for the perturbative amplitudes, as will be discussed below. We remark that all the terms in $T_{4}^{\text {uni }}$ will be proportional to the $\bar{J}$ and $\overline{\bar{J}}$ functions defined in Appendix A. 
Using crossing symmetry it is not difficult to see that there are only eight independent meson-meson amplitudes. We have calculated these amplitudes to one loop in SU(3) ChPT. They are given in Appendix B. Three of these amplitudes had not been calculated before, namely, $\bar{K}^{0} \eta \rightarrow \bar{K}^{0} \eta$, $\eta \eta \rightarrow \eta \eta$, and $K \eta \rightarrow K \pi^{0}$. For the rest, we have checked that our amplitudes coincide with previous results $[10,11]$ up to differences in notation and different simplification schemes, equivalent up to $O\left(p^{4}\right)$. In particular, since we are interested in the "exact" form of perturbative unitarity (see below), we have written our final results in terms of a single pion decay constant $f_{\pi}$, and we have used the Gell-MannOkubo relation, taking care to preserve exact perturbative unitarity. Furthermore, we have explicitly checked that all the amplitudes remain finite and scale independent.

Finally, we wish to add a remark about $\eta$ - $\eta^{\prime}$ mixing, since the physical $\eta$ is indeed a mixture of the U(3) octet and singlet pseudoscalars, whereas in this work we are only using the standard SU(3) ChPT. One may wonder then if our description of the $\eta$ is just that of the pseudoscalar octet component, since in this Lagrangian the singlet field is not an explicit degree of freedom. However, it has been shown [13] that the standard framework results from an expansion in powers of the inverse powers of the "topological susceptibility" of the complete U(3) Lagrangian. In that context the $\eta^{\prime}$ is considered as a massive state (that is why it does not count as an explicit degree of freedom) but the singlet component generates a correction to $L_{7}$. Note that, indeed, the mass of the $\eta$ contains an $L_{7}$ contribution, and that is why we can use $M_{\eta}$ in Eq. (8) with its physical value, whereas $M_{0 \eta}$ is the one satisfying the Gell-Mann-Okubo relation exactly. Therefore, our approach can be understood as the lowest order approximation to the $\eta$ - $\eta^{\prime}$ mixing problem, where all the effects of the mixing appear only through $L_{7}$. Since we will compare only with data in states with one $\eta$ at most, and below $1200 \mathrm{MeV}$, our results seem to suggest that this approximation, although somewhat crude, is enough with the present status of the experimental data. Indeed, we will see that the values that we obtain for $L_{7}$ are in perfect agreement with those given in the literature (and this comparison can now be done because we have the complete one-loop amplitudes renormalized in the standard way).

\section{PARTIAL WAVES AND UNITARITY}

\section{A. Partial waves}

Let us denote by $T_{a b}^{I J}(s)$ the partial wave for the process $a \rightarrow b$, i.e., the projection of the amplitude for that process with given total isospin $I$ and angular momentum $J$. That is, if $T_{a b}^{I}(s, t, u)$ is the isospin combination with total isospin $I$, one has

$$
T_{a b}^{I J}(s)=\frac{1}{32 N \pi} \int_{-1}^{1} d x P_{J}(x) T_{a b}^{I}(s, t(s, x), u(s, x))
$$

where $t(s, x), u(s, x)$ are given by the kinematics of the process $a \longrightarrow b$ with $x=\cos \theta$, the scattering angle in the center of mass frame.
Note that we are normalizing the partial waves including a factor $N$, such that $N=2$ if all the particles in the process are identical and $N=1$ otherwise. Recall that, since we are working in the isospin limit, the three pions are considered as identical, so that $N=2$ only for the $\pi \pi \rightarrow \pi \pi$ and $\eta \eta$ $\rightarrow \eta \eta$ processes.

We shall comment now on the $T_{a b}^{I}$ amplitudes for every possible process involving $\pi, K, \eta$. Using crossing symmetry and assuming isospin symmetry exactly, we will determine the number of independent amplitudes for each process. The discussion is general and there is no need to invoke ChPT, although we will refer to the results for the amplitudes in Appendix B, which gives the one-loop ChPT results.

$\pi \pi \rightarrow \pi \pi$ scattering. There is only one independent amplitude, so that one has

$$
\begin{gathered}
T^{0}(s, t, u)=3 T(s, t, u)+T(t, s, u)+T(u, t, s), \\
T^{1}(s, t, u)=T(t, s, u)-T(u, t, s), \\
T^{2}(s, t, u)=T(t, s, u)+T(u, t, s),
\end{gathered}
$$

where $T(s, t, u)$ is the $\pi^{+} \pi^{-} \rightarrow \pi^{0} \pi^{0}$ amplitude. At one loop in ChPT it is given in Appendix B, Eq. (B4).

$K \pi \rightarrow K \pi$ scattering. Crossing symmetry allows us to write the $I=1 / 2$ in terms of the $I=3 / 2$ one as

$$
T^{1 / 2}(s, t, u)=\frac{1}{2}\left[3 T^{3 / 2}(u, t, s)-T^{3 / 2}(s, t, u)\right] .
$$

Here, $T^{3 / 2}(s, t, u)$ is the $K^{+} \pi^{+} \rightarrow K^{+} \pi^{+}$amplitude, whose expression at one loop within ChPT corresponds to Eq. (B5).

$K \bar{K} \rightarrow K \bar{K}$ scattering. We can write the isospin amplitudes as

$$
\begin{aligned}
& T^{0}(s, t, u)=T_{\mathrm{ch}}(s, t, u)+T_{\text {neu }}(s, t, u), \\
& T^{1}(s, t, u)=T_{\mathrm{ch}}(s, t, u)-T_{\text {neu }}(s, t, u),
\end{aligned}
$$

where $T_{\text {ch }}$ and $T_{\text {neu }}$ are, respectively, the amplitudes for the processes $K^{+} K^{-} \rightarrow K^{+} K^{-}$and $\bar{K}^{0} K^{0} \rightarrow K^{+} K^{-}$. Their expressions to one loop correspond to Eqs. (B7) and (B8), respectively.

$K \bar{K} \rightarrow \pi \pi$ scattering. In this case, one has

$$
\begin{aligned}
& T^{0}(s, t, u)=\frac{\sqrt{3}}{2}\left[T^{3 / 2}(u, s, t)+T^{3 / 2}(t, s, u)\right], \\
& T^{1}(s, t, u)=\frac{1}{\sqrt{2}}\left[T^{3 / 2}(u, s, t)-T^{3 / 2}(t, s, u)\right],
\end{aligned}
$$

where $T^{3 / 2}(s, t, u)$ is the $K^{+} \pi^{+} \rightarrow K^{+} \pi^{+}$amplitude, given in Appendix B for one-loop ChPT, Eq. (B5).

$K \eta \rightarrow K \eta$ scattering. This is a pure $I=1 / 2$ process. The one-loop amplitude can be read directly from Eq. (B2).

$\bar{K} K \rightarrow \eta \eta$ scattering. This is an $I=0$ process that using crossing symmetry can be obtained from the previous amplitude as follows:

$$
T_{\bar{K}^{0} K^{0} \rightarrow \eta \eta}(s, t, u)=T_{K^{0} \eta \rightarrow \bar{K}^{0} \eta}(t, s, u) .
$$


$K \eta \rightarrow K \pi$ scattering. This is also an $I=1 / 2$ process, whose amplitude, correctly normalized, is

$$
T^{1 / 2}(s, t, u)=-\sqrt{3} T_{\bar{K}^{0} \eta \rightarrow \bar{K}^{0} \pi^{0}(s, t, u),}
$$

where the one-loop expression for $\bar{K}^{0} \eta \rightarrow \bar{K}^{0} \pi^{0}$ can be found in Eq. (B3).

$\bar{K} K \rightarrow \pi \eta$ scattering. This is an $I=1$ process related to the $\bar{K}^{0} \eta \rightarrow \bar{K}^{0} \pi^{0}$ amplitude by crossing symmetry, i.e.,

$$
T^{1}(s, t, u)=-\sqrt{2} T_{\bar{K}^{0} \eta \rightarrow \bar{K}^{0} \pi^{0}}(t, s, u) .
$$

$\pi \eta \rightarrow \pi \eta$ scattering. This is a pure $I=1$ isospin amplitude whose one-loop ChPT expression can be read directly from Eq. (B6).

$\pi \pi \rightarrow \eta \eta$ scattering. Now $I=0$ and the amplitude is obtained from the previous one by crossing, as

$$
T_{\pi^{0} \pi^{0} \rightarrow \eta \eta}(s, t, u)=T_{\pi^{0} \eta \rightarrow \pi^{0} \eta}(t, s, u) .
$$

$\eta \eta \rightarrow \eta \eta$ scattering. Here, $I=0$ and the corresponding one-loop amplitude can also be read directly from Eq. (B1).

In this paper we will be interested in the case when there are several coupled states for a given choice of $I, J$, i.e., the coupled channel case. In particular, with the above normalization, the relationship between the $T$-matrix elements $T_{a b}^{I J}$ and the $S$-matrix ones is given for two coupled channels $(a, b=1,2)$ by

$$
\begin{gathered}
S_{11}=1+2 i \sigma_{1} T_{11}, \\
S_{22}=1+2 i \sigma_{2} T_{22}, \\
S_{12}=S_{21}=2 i \sqrt{\sigma_{1} \sigma_{2}} T_{12},
\end{gathered}
$$

where the $I J$ superscripts have been suppressed to ease the notation and we have used the fact that due to time reversal invariance $T_{i j}=T_{j i}$. Here, $\sigma_{i}=2 q_{i} / \sqrt{s}$ where $q_{i}$ is the center of mass momentum in the state $i$. Note that $\sigma_{i}$ is nothing but the phase space of that state at $\sqrt{s}$. In the $I=0$ channel above the $\eta \eta$ threshold we will use the corresponding generalization in the case of three channels.

\section{B. Unitarity}

The $S$ matrix should be unitary, i.e., $S S^{\dagger}=1$. In case there is only one state available, that means that $S$ can be parametrized in terms of a single observable, which is customarily chosen as the phase shift. For the case of two channels, the elements $S_{i j}$ are organized in a unitary $2 \times 2$ matrix, containing only three independent parameters. We will follow the standard parametrization:

$$
S=\left(\begin{array}{cc}
\eta e^{2 i \delta_{1}} & i \sqrt{1-\eta^{2}} e^{i\left(\delta_{1}+\delta_{2}\right)} \\
i \sqrt{1-\eta^{2}} e^{i\left(\delta_{1}+\delta_{2}\right)} & \eta e^{2 i \delta_{2}}
\end{array}\right),
$$

where the $\delta_{i}$ are the phase shifts and $\eta$ is the inelasticity.

The unitarity relation translates into relations for the elements of the $T$ matrix of a particularly simple form for the partial waves. For instance, if there is only one possible state
1 for a given choice of $I, J$, the partial wave $T_{11}$ satisfies Eq. (19), so that unitarity means that

$$
\operatorname{Im} T_{11}=\sigma_{1}\left|T_{11}\right|^{2} \Rightarrow \operatorname{Im} T_{11}^{-1}=-\sigma_{1} .
$$

In principle, the above equation holds only above threshold up to the energy where another state 2 is physically accessible. If there are two states available, then the $T$-matrix elements satisfy

$$
\begin{gathered}
\operatorname{Im} T_{11}=\sigma_{1}\left|T_{11}\right|^{2}+\sigma_{2}\left|T_{12}\right|^{2}, \\
\operatorname{Im} T_{12}=\sigma_{1} T_{11} T_{12}^{*}+\sigma_{2} T_{12} T_{22}^{*}, \\
\operatorname{Im} T_{22}=\sigma_{1}\left|T_{12}\right|^{2}+\sigma_{2}\left|T_{22}\right|^{2} .
\end{gathered}
$$

In matrix form they read

$$
\operatorname{Im} T=T \Sigma T^{*} \Rightarrow \operatorname{Im} T^{-1}=-\Sigma,
$$

with

$$
T=\left(\begin{array}{ll}
T_{11} & T_{12} \\
T_{12} & T_{22}
\end{array}\right), \quad \Sigma=\left(\begin{array}{cc}
\sigma_{1} & 0 \\
0 & \sigma_{2}
\end{array}\right),
$$

which allows for a straightforward generalization to the case of $n$ accessible states by using $n \times n$ matrices.

One must bear in mind that the unitarity relations imply that the partial waves are bounded as the energy increases. For instance, in the one-channel case, from Eq. (23) we can write

$$
T_{11}=\frac{\sin \delta}{\sigma_{1}} e^{i \delta}
$$

where $\delta$ is the phase of $t_{11}$.

Note that all the unitarity relations Eqs. (23) and (24) are linear on the left hand side and quadratic on the right. As a consequence, if one calculates the amplitudes perturbatively as truncated series in powers of an expansion parameter, say $T=T_{2}+T_{4}+\cdots$, the unitarity equations will never be satisfied exactly. In particular, for ChPT that means that unitarity can only be satisfied perturbatively: i.e.,

$$
\begin{gathered}
\operatorname{Im} T_{2}=0, \\
\operatorname{Im} T_{4}=T_{2} \Sigma T_{2}, \\
\cdots,
\end{gathered}
$$

where the second equation is satisfied exactly only if one is careful to express $T_{4}$ in terms of masses and decay constants consistently with the choice made for $T_{2}$. That has not always been the case in the literature and that is one of the reasons why we have recalculated some processes: all our results satisfy exact perturbative unitarity. Otherwise there are additional $O\left(p^{6}\right)$ terms in Eq. (27). As we will see below, this will be relevant in obtaining a simple formula for the unitarized amplitudes. Our choice has been to rewrite all the $f_{K}$ and $f_{\eta}$ contained in the amplitudes in terms of $f_{\pi}, L_{4}^{r}$, and $L_{5}^{r}$ using the relations in Eq. (9). 
The deviations from Eq. (24) are more severe at high energies, and in particular in the resonance region, since unitarity implies that the partial waves are bounded [see Eq. (26)], which cannot be satisfied by a polynomial. Generically, in the resonance region, the unitarity bounds are saturated. If a polynomial is adjusted to saturate unitarity in a given region, in general, it will break the unitarity bound right afterward. Another way of putting it is that resonances are associated with poles in the complex plane, which will never be reproduced with polynomials.

For all these reasons, if we are interested in extending the good properties of ChPT to higher energies, we have to modify the amplitudes, imposing unitarity and a functional form that allows for poles in the complex plane. This will be achieved with the inverse amplitude method.

\section{THE COUPLED CHANNEL INVERSE AMPLITUDE METHOD}

As can be seen from the unitarity condition in Eq. (24), the imaginary part of the inverse amplitude is known exactly above the corresponding thresholds, namely, $\operatorname{Im} T^{-1}=-\Sigma$. Indeed, any amplitude satisfying the unitarity constraint should have the following form:

$$
T=\left(\operatorname{Re} T^{-1}-i \Sigma\right)^{-1},
$$

Consequently, we should only have to calculate the real part of $T^{-1}$. As a matter of fact, many unitarization methods are just different approximations to $\operatorname{Re} T^{-1}$ (see [9] for details). The idea behind the inverse amplitude method is to use Eq. (28), but approximating $\operatorname{Re} T^{-1}$ with ChPT. Since we have $T \simeq T_{2}+T_{4}+\cdots$, then

$$
\begin{aligned}
T^{-1} & \simeq T_{2}^{-1}\left(1-T_{4} T_{2}^{-1}+\cdots\right), \\
\operatorname{Re} T^{-1} & \simeq T_{2}^{-1}\left[1-\left(\operatorname{Re} T_{4}\right) T_{2}^{-1}+\cdots\right],
\end{aligned}
$$

so that multiplying Eq. (28) by $T_{2} T_{2}^{-1}$ on the left and $T_{2}^{-1} T_{2}$ on the right, we find

$$
T \simeq T_{2}\left(T_{2}-\operatorname{Re} T_{4}-i T_{2} \Sigma T_{2}\right)^{-1} T_{2} .
$$

At this point, if the amplitude satisfies "exact perturbative unitarity," namely, Eq. (27), we can simplify the above equation to obtain the simple expression

$$
T \simeq T_{2}\left(T_{2}-T_{4}\right)^{-1} T_{2} .
$$

This is the generalization of the IAM to coupled channels. Note that this formula ensures exact unitarity only if $T_{4}$ satisfies exact perturbative unitarity.

The IAM was first applied to just one elastic channel [7] and it was able to reproduce well the $\pi \pi$ and $\pi K$ scattering phase shifts below the $K \bar{K}$ and $K \eta$ thresholds, respectively. In addition it was able to generate the $\sigma$ [now called $\left.f_{0}(400-1200)\right]$, the $\rho$, and the $K^{*}$ resonances [8]. Furthermore, it was shown how the formula for the one-channel IAM can be justified in terms of dispersion relations [8], which allowed for the analytic continuation to the complex plane and the identification of the pole associated with each resonance in the second Riemann sheet.

In view of Eq. (32), it may seem necessary to know the complete $O\left(p^{4}\right)$ ChPT calculation of each one of the $T$-matrix elements. Nevertheless, one could use a further approximation and calculate only the $s$-channel loops (Fig. 1a), which are the only ones responsible for the unitarity cut and are supposed to dominate in the resonant region. This was the approach followed in [9], having in mind that the complete ChPT calculations were not available at that time for any meson-meson scattering two-channel matrix. The results were remarkable, reproducing up to $1.2 \mathrm{GeV}$ seven $(I, J)$ meson-meson scattering channels (17 amplitudes), and even generating seven resonances. However, the fact that the $s$-channel loops were regularized with a cutoff, together with the omission of crossed loops and tadpoles, made it impossible to compare the chiral parameters with those of standard ChPT (still, they had the correct order of magnitude, as expected). In addition, the low energy ChPT predictions were recovered only partially. This motivated the authors in [11] to calculate the full $O\left(p^{4}\right) \quad K^{+} K^{-} \rightarrow K^{+} K^{-}$and $K^{+} K^{-}$ $\rightarrow K^{0} \bar{K}^{0}$ amplitudes, ${ }^{1}$ which allowed for the unitarization with Eq. (32) of the $(I, J)=(0,0)$ and $(1,1)$ channels. This approach again yielded a good high energy description but also reproduced simultaneously the low energy $\pi \pi$ scattering lengths. All these results were obtained with $L_{i}$ parameters compatible with those of standard ChPT [11].

As we saw in the previous section, we have calculated the last three independent $O\left(p^{4}\right)$ meson-meson scattering amplitudes that were still missing. They are given in Appendix B in a unified notation with the other five that we have recalculated independently (correcting some minor misprints in the literature). Therefore, we are now ready to unitarize the complete meson-meson scattering by means of Eq. (32).

However, at this point we have to recall that for a given energy Eq. (32) has been justified only for a matrix whose dimension is exactly the number of states accessible at that energy. The reason is that the unitarity relation Eq. (24) increases its dimensionality each time we cross a new threshold. Thus, for instance, in $\pi \pi$ scattering, one should use the one-dimensional IAM up to the $K \bar{K}$ threshold, then the twodimensional IAM, etc., although this procedure yields discontinuities on each threshold, instead of a single analytic function. Another possibility [9] is to use the IAM with the highest possible dimensionality of the $I, J$ channel for all energies. ${ }^{2}$ This second possibility yields an analytic (and

\footnotetext{
${ }^{1}$ An erratum for these amplitudes has appeared, published when we were preparing this work. The previous results and conclusions in [11] are nevertheless correct, since the errata did not affect the numerical calculations. We thank J. A. Oller for discussions and for letting us check that their corrected amplitudes coincide with ours.

${ }^{2}$ As a technical remark, let us note that in this case the IAM has to be rederived in terms of the partial waves $T_{a b}$ divided by the c.m. momenta of the initial and final states to the $J$ th power, to ensure that these new amplitudes are real at lowest order. From there the derivation follows the same steps, and we recover the very same Eq. (32) by multiplying by the initial and final state momenta in the end.
} 
hence continuous) function, but it may not satisfy unitarity exactly at all energies, namely, when the number of opened channels is smaller than the dimensionality of the IAM formula. Following the $\pi \pi-K \bar{K}$ example, if we use the twodimensional IAM formula, we will have exact unitarity ensured above the $K \bar{K}$ threshold, but not below. In particular, if we still use the two-dimensional IAM below the $K \bar{K}$ threshold, the IAM $\pi \pi$ scattering element will have an additional spurious contribution from the imaginary part of the $K \bar{K}$ scattering left cut, which extends up to $\sqrt{s}=4\left(M_{K}^{2}-M_{\pi}^{2}\right)$. This is a well known and lasting problem in the literature [14-16] that affects other unitarization methods also, like the $K$-matrix method [15]. As a matter of fact, several years ago [14] it was suggested that the physical solution would probably be an interpolation between the two approaches just mentioned. However, in the context of ChPT and the IAM, and for the $\pi \pi-K \bar{K}$ channels, it was found [11] that the violations of unitarity are, generically, of the order of a few percent only. We have confirmed this result but now for the whole meson-meson scattering sector. Even the threshold parameters can be accurately reproduced, since they are defined through the real parts of the amplitudes, which are almost not affected by the spurious part. The origin of this problem is that the IAM in Eq. (32) mixes the left cuts of all the channels involved when performing the inverse of the $T_{2}-T_{4}$ matrix. Thus, it is not able to reproduce the left cut singularities correctly [8,17], although numerically their contribution is negligible when all the observables are expressed in terms of the real parts of the amplitude, and taking into account the present status of the data and the uncertainties in the $L_{i}$.

In this paper we have chosen to show the second approach, since the one-dimensional IAM has been thoroughly studied in [8]. Very recently, there have been dispersive approaches [16] proposed to circumvent this problem in the $\pi \pi-\bar{K} K$ system, but they involve the calculation of left cut integrals that are hard to estimate theoretically. It would be interesting to have them extended and related to the ChPT formalism, but that is beyond the scope of this work. The fact that we use the higher dimensional IAM formalism, which contains spurious cuts, does not allow for a clean continuation to the complex plane. Nevertheless, since poles associated with resonances have already been found in the onedimensional case [8] and in other approximated coupled channel IAM approaches [9], we leave their description for a generalized IAM approach with better analytic properties [18]. In this work we will concentrate on physical $s$ values, and the compatibility of the unitarized description of resonances and low energy data with existing determinations of the chiral parameters. Nevertheless, we will also show that this can also be achieved with the first, discontinuous, approach.

\section{MESON-MESON SCATTERING DATA}

Let us then comment on the data available for each channel.

\section{Channel $(I, J)=(1,1)$}

For the energies considered here, the two states that may appear in this channel are $\pi \pi$ and $K \bar{K}$. In Figs. 2(a) and 3(a), we plot the data on the $\pi \pi$ scattering phase shift obtained from [19] and [20], which correspond to the squares and triangles, respectively. Let us remark that the first set of data points tends to be between two and three standard deviations higher than the second when the phase shift is higher than $90^{\circ}$, and the other way around for smaller values of the phase shift (note that the error bars are smaller than the data symbols). Thus the data sets are not quite consistent with one another, which could be fixed with the addition of a systematic error of the order of a few percent.

This channel is completely dominated by the $\pi \pi$ state and there is almost no inelasticity due to $K \bar{K}$ production below $1200 \mathrm{MeV}$. The $\left(1-\eta_{11}^{2}\right) / 4$ points from the inelasticity analysis given in [21] are shown in the lowest part of Figs. 2(d) and 3(d).

\section{Channel $(I, J)=(0,0)$}

For this channel we may have up to three states, namely, $\pi \pi, K \bar{K}$ and $\eta \eta$. In this case, there are three observables with several sets of data, which, as can be seen in Figs. 2(b), 2(c), and 2(d), are somewhat incompatible between themselves when considering only the errors quoted in the experiments. Again, they become compatible if we assume a systematic error of a few percent. For the $\pi \pi$ scattering phase shift $\left(\delta_{00}\right.$, see Fig. 2(b)), the experimental data shown come from different analyses of the CERN-Munich Collaboration [22] (open squares), as well as from [19] (solid squares), [23] (solid triangles), and [24] (solid circles). Concerning the $\pi \pi \rightarrow K \bar{K}$ phase shift, the data in Figs. 2(c) and 3(c) correspond to [21] (solid triangles) and [25] (solid squares) and they are reasonably compatible, mainly due to the large errors in the first set. Finally, we also show in Figs. 2(d) and $3(\mathrm{~d})$ the data for $\left(1-\eta_{00}^{2}\right) / 4$, since it is customary to represent in that way the values of the inelasticity $\eta_{00}$. The experimental results are rather confusing here, mainly up to $1100 \mathrm{MeV}$, due to problems in the normalization. From the data shown in the figure, in Sec. VII we have only fitted to those coming from [25] (solid squares), [26] (solid triangles), [27] (open squares), and [28] (open circles). There is a disagreement in the normalization of the data of [29] (diamonds) up to a factor of 2 (see [30] for a discussion). We have not included the latter in the fit, mostly because in the analysis of [29] the authors neglect the unitarity constraint, which in our approach is satisfied exactly at those energies.

\section{Channel $(I, J)=(2,0)$}

There is only the $\pi \pi$ state and so we display in Figs. 2(e) and 3(e) only the $\delta_{20}$ phase shifts again from the CERNMunich Collaboration [31] (open squares) and the CERNSaclay Collaboration [32] (solid triangles).

\section{Low energy $K_{l 4}$ decay data}

This reaction is particularly important since it yields very precise information on the $\delta_{00^{-}} \delta_{11}$ combination of $\pi \pi$ scat- 

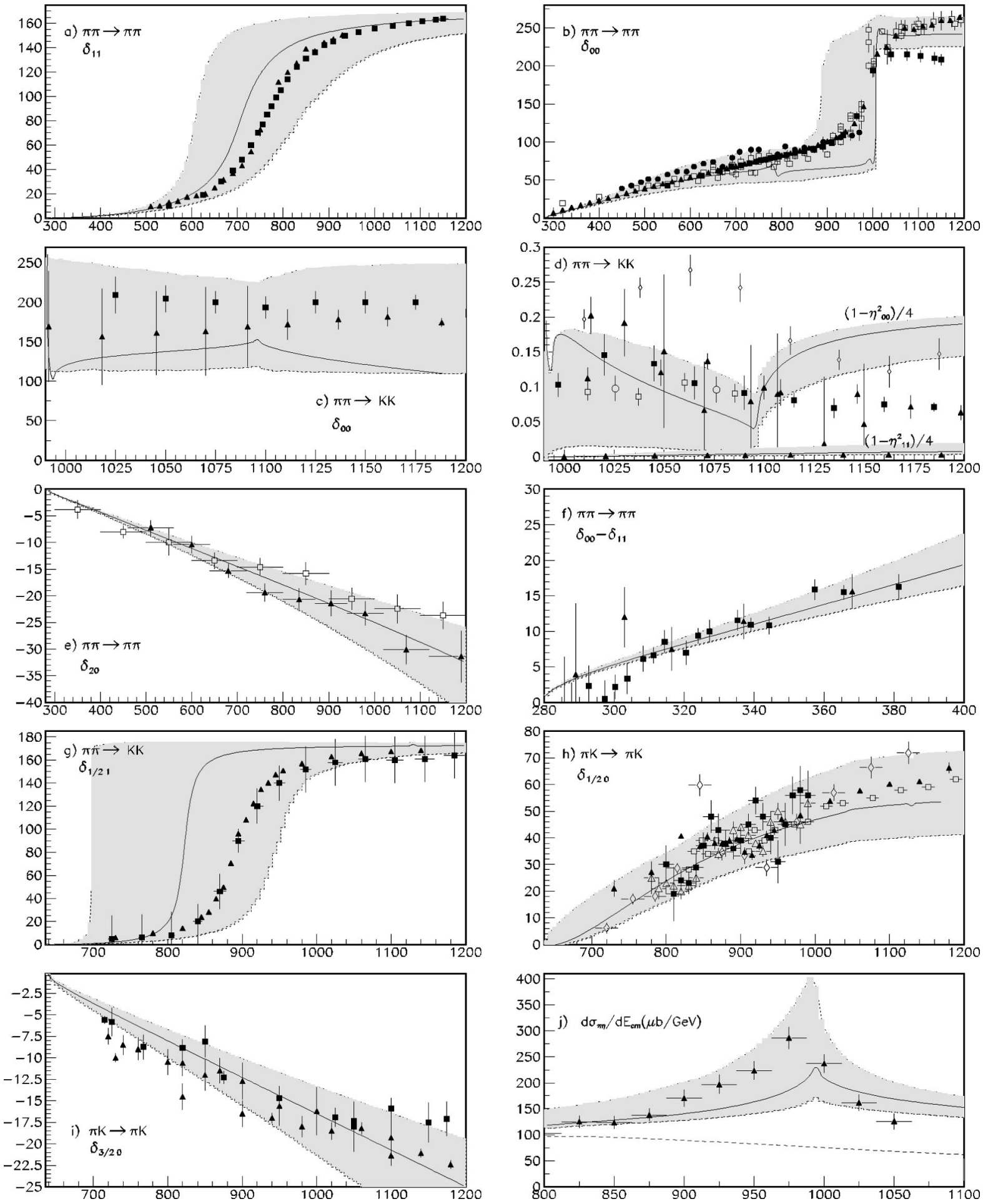

FIG. 2. The curves represent the result of applying the coupled channel IAM using the determination of the ChPT low energy constant given in the fourth column of Table I. The shaded area covers the uncertainty due to the errors in those determinations (assuming they were totally uncorrelated).

tering phase shifts at very low energies. In Figs. 2(f) and 3(f) we show the data from the Geneva-Saclay group [33] (solid triangles) and the very recent, and more precise, data from the E865 Collaboration at Brookhaven [34] (solid squares).
Channel $(I, J)=(1 / 2,1)$

Here the possible states are $K \pi$ and $K \eta$. We have plotted in Figs. 2(g) and $3(\mathrm{~g})$ data from the following experiments: [35] (solid squares) and [36] (solid triangles). Note 

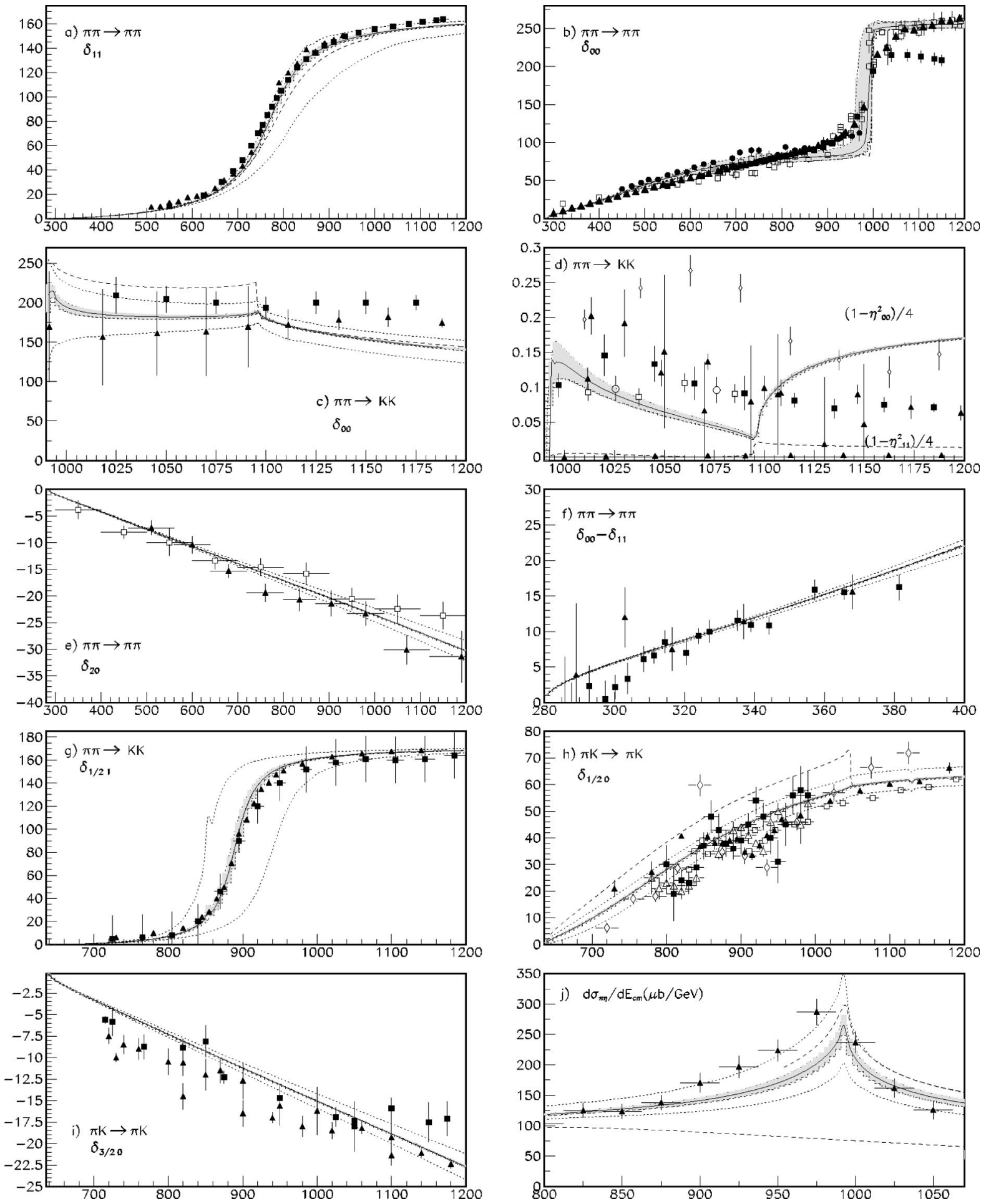

FIG. 3. The curves represent the result of the coupled channel IAM fit to meson-meson scattering observables that is described in the text. The shaded area covers only the uncertainty due to the statistical errors in the $L_{i}$ parameters obtained from MINUIT (assuming they were uncorrelated). The area between the dotted lines corresponds to the error bands including in the $L_{i}$ the systematic error added to the data (see text for details). Finally, the dashed line corresponds to the use of the one-channel IAM when only one channel is accessible, but keeping the same parameters as in the previous fit.

that the first set is systematically lower than the second, which is newer and more precise. Nevertheless, they are compatible, thanks mostly to the large error bars on the first set.

\section{Channel $(I, J)=(1 / 2,0)$}

Here the states are also $K \pi$ and $K \eta$. The data in Figs. 2(h) and 3(h) come from the following experiments: [35] (solid squares), [37] (open triangles), [38] (open diamonds), 
[36] (solid triangles), and [39] (open squares). It can be easily noticed that not all the data sets are compatible within errors, but once again they can be reconciled by assuming a systematic error of the order of a few percent.

\section{Channel $(I, J)=(3 / 2,0)$}

The only state here is $\pi K$. In this case we have plotted in Figs. 2(i) and 3(i) data sets from [36] (solid triangles) and [40] (solid squares). The latter are somewhat lower than the former, although they are compatible mostly due to the large errors in [36].

\section{Channel $(I, J)=(1,0)$}

The possible states for this case are $\pi \eta$ and $K \bar{K}$. We have plotted in Figs. 2(j) and 3(j) the $\pi \eta$ effective mass distribution from the $p p \rightarrow p\left(\eta \pi^{+} \pi^{-}\right) p$ reaction studied by the WA76 Collaboration [41]. In order to reproduce these data, we use

$$
\frac{d \sigma_{\pi \eta}}{d E_{\mathrm{c} . \mathrm{m} .}}=c p_{\pi \eta}\left|T_{12}^{10}\right|^{2}+\text { background, }
$$

where the $c$ factor accounts for the normalization of the mass distribution and the dashed curve in these figures corresponds to a background due to other resonances apart from the $a_{0}(980)$ (see [41] for details).

Once we have described the data in the different channels, we will first compare with the IAM "predictions" from the present values of the ChPT low energy constants, and later we will fit these data by means of the IAM.

\section{THE IAM WITH PRESENT LOW ENERGY CONSTANT DETERMINATIONS}

In this section we will comment on the results of applying the coupled channel IAM using the low energy constants from standard ChPT. Since the values of these constants have been determined from low energy data or large $N_{c}$ arguments, the high energy results could be considered as predictions of the IAM. For our calculations we have used $f_{\pi}$ $=92.4 \mathrm{MeV}, M_{\pi}=139.57 \mathrm{MeV}, M_{K}=495.7 \mathrm{MeV}$, and $M_{\eta}$ $=547.45 \mathrm{MeV}$.

In the second column of Table I we list the values obtained from a very recent and precise two-loop $O\left(p^{6}\right)$ analysis of $K_{l 4}$ decays [42]. Note that the errors are only statistical. In the next column we list the central values of the same analysis but only at $O\left(p^{4}\right)$. In the fourth column we list the values from another set where $L_{1}, L_{2}, L_{3}$ are taken from an overall fit to $K_{e 4}$ and $\pi \pi$ data [43] and the rest are taken from [2]. Note that all of them are quite compatible and, except for $L_{5}$, the size of the error bars is comparable.

In Fig. 2 we show the results of the IAM with the values given in the fourth column of Table I. The solid curve corresponds to the central values, whereas the shaded areas cover the uncertainty due to the error on the parameters. They have been obtained with a Monte Carlo Gaussian sampling of 1000 choices of low energy constants for each $\sqrt{s}$, assuming the errors are uncorrelated. It is worth noticing that
TABLE I. Different sets of chiral parameters $\times 10^{3}$. The second and third columns come from an $O\left(p^{6}\right)$ and $O\left(p^{4}\right)$ analysis of $K_{l 4}$ decays [42], respectively. Note that $L_{4}^{r}$ and $L_{6}^{4}$ are set to zero. In the third column $L_{1}^{r}, L_{2}^{r}, L_{3}$ are taken from [43] and the rest from [2] ( $L_{4}^{r}$ and $L_{6}^{r}$ are estimated from the Zweig rule).

\begin{tabular}{cccc}
\hline \hline & $K_{l 4}$ decays $O\left(p^{6}\right)$ & $K_{l 4}$ decays $O\left(p^{4}\right)$ & ChPT \\
\hline$L_{1}^{r}\left(M_{\rho}\right)$ & $0.53 \pm 0.25$ & 0.46 & $0.4 \pm 0.3$ \\
$L_{2}^{r}\left(M_{\rho}\right)$ & $0.71 \pm 0.27$ & 1.49 & $1.35 \pm 0.3$ \\
$L_{3}$ & $-2.72 \pm 1.12$ & -3.18 & $-3.5 \pm 1.1$ \\
$L_{4}^{r}\left(M_{\rho}\right)$ & 0 & 0 & $-0.3 \pm 0.5$ \\
$L_{5}^{r}\left(M_{\rho}\right)$ & $0.91 \pm 0.15$ & 1.46 & $1.4 \pm 0.5$ \\
$L_{6}^{r}\left(M_{\rho}\right)$ & 0 & 0 & $-0.2 \pm 0.3$ \\
$L_{7}$ & $-0.32 \pm 0.15$ & -0.49 & $-0.4 \pm 0.2$ \\
$L_{8}^{r}\left(M_{\rho}\right)$ & $0.62 \pm 0.2$ & 1.00 & $0.9 \pm 0.3$ \\
\hline \hline
\end{tabular}

these error bands are so wide that the results for the other columns in Table I are rather similar, even for the central values. Qualitatively all of them look the same.

It is noticeable that the IAM results, even with the low energy parameters from standard ChPT, already provide distinct resonant shapes of the $\rho, f_{0}(980), K^{*}$, and $a_{0}(980)$ (see Figs. 2(a), 2(b), 2(g), and 2(j), respectively). In addition, the IAM also provides two other extremely wide structures in the $(0,0) \pi \pi$ and $(1 / 2,0) \pi K$ scattering amplitudes. They correspond to the $\sigma$ [or $\left.f_{0}(400-1200)\right]$ and $\kappa$ (see Figs. 2(b) and 2(h)). These structures are too wide to be considered as Breit-Wigner resonances, but they are responsible for the relatively high values of the phase shifts (the strength of the interaction) already near threshold. In recent years there has been a considerable discussion about the existence and properties of these two states (for references, see the scalar meson review by the Particle Data Group (PDG) [45]). Since ChPT does not deal directly with quarks and gluons, it is very difficult to make any conclusive statement about the spectroscopic nature of these states (whether they are $q \bar{q}$, fourquark states, meson molecules, etc.) unless we make additional assumptions [44], which would then spoil much of the model independency of our approach, which is based just on chiral symmetry and unitarity. Nevertheless, the simplicity and remarkable results of this method give strong support, from the theoretical side, for the existence of both the $\sigma$ and the $\kappa$ poles. From previous work, it is known that the ChPT amplitudes unitarized with the IAM generate the poles in the second Reimann sheet associated with the $\sigma$ and the $\kappa$ around $\sqrt{s_{\text {pole }}} \simeq 440-i 225[8,9]$ and $770-i 250 \mathrm{MeV}[9]$, respectively. (Let us remember that, since these states are very wide, the familiar relations $M \simeq \operatorname{Re} \sqrt{s_{\text {pole }}}$ and $\Gamma \simeq$ $-2 \operatorname{Im} \sqrt{s_{\text {pole }}}$ are very crude approximations.) We have checked that similar results are obtained for the amplitudes of this work. These values have to be considered as estimates, since the uncertainties must be rather big, taking into account that the data in these channels are very conflicting (see Figs. 2 and 3). The fact that we are able to reproduce these states with parameters compatible with previous determinations is also strong support for their pole positions, which are in agreement with recent experimental determina- 
TABLE II. Low energy constants $\left(\times 10^{3}\right)$ obtained from an IAM fit to the meson-meson scattering data. The errors listed in the second column are obtained by adding in quadrature those of columns 3 and 4 .

\begin{tabular}{cccc}
\hline \hline & $\begin{array}{c}\text { Fit+ errors } \\
\text { (curve in Fig. 3) }\end{array}$ & $\begin{array}{c}\text { MINUIT error } \\
\text { (band in Fig. 3) }\end{array}$ & $\begin{array}{c}\text { Systematic error } \\
\text { from data }\end{array}$ \\
\hline$L_{1}^{r}\left(M_{\rho}\right)$ & $0.56 \pm 0.10$ & \pm 0.008 & \pm 0.10 \\
$L_{2}^{r}\left(M_{\rho}\right)$ & $1.21 \pm 0.10$ & \pm 0.001 & \pm 0.10 \\
$L_{3}$ & $-2.79 \pm 0.14$ & \pm 0.02 & \pm 0.12 \\
$L_{4}^{r}\left(M_{\rho}\right)$ & $-0.36 \pm 0.17$ & \pm 0.02 & \pm 0.17 \\
$L_{5}^{r}\left(M_{\rho}\right)$ & $1.4 \pm 0.5$ & \pm 0.02 & \pm 0.5 \\
$L_{6}^{r}\left(M_{\rho}\right)$ & $0.07 \pm 0.08$ & \pm 0.03 & \pm 0.08 \\
$L_{7}$ & $-0.44 \pm 0.15$ & \pm 0.003 & \pm 0.15 \\
$L_{8}^{r}\left(M_{\rho}\right)$ & $0.78 \pm 0.18$ & \pm 0.02 & \pm 0.18 \\
\hline
\end{tabular}

tions for both the $\sigma$ and the $\kappa$ [46].

To summarize, we have just shown how the present status of both the experimental data and the $L_{i}$ determinations allows for use of the IAM despite the approximations made in its derivation, like the poor description of the left cut noted above.

\section{INVERSE AMPLITUDE METHOD FIT TO THE SCATTERING DATA}

Once we have seen that the IAM already describes the basic features of meson-meson scattering, we can proceed to fit the data in order to obtain a more accurate description. For that purpose we have used the MINUIT function minimization and error analysis routine from the CERN program library [47].

Our results are presented in Fig. 3, whose different curves and bands can be understood as follows. As we have already seen when commenting on the experiments in the previous section, and as can be observed in Figs. 2 and 3, there are several incompatible sets of data for some channels. In the literature, this is usually solved by adding an extra systematic error until these values are compatible. We have made three fits by adding $1 \%, 3 \%$, and $5 \%$ errors to the data in each channel. The continuous line corresponds to the $3 \%$ case and the resulting $L_{i}$ values are listed in the second column of Table II. The shaded areas have been obtained again from a Monte Carlo sampling using the $L_{i}$ uncertainties given by MINUIT for this fit, which are listed in the third column of Table II. Let us remark that there would be almost no difference to the naked eye if we showed the fit with a $1 \%$ or a $5 \%$ error, in either the central continuous line or the shaded bands. Furthermore, the $\chi^{2}$ per degree of freedom for any of these fits is always $O(1)$.

However, although the curves remain almost unchanged when fitting with a different global systematic error, the values of $L_{i}$ come out somewhat differently from each fit. This is an additional source of error on the $L_{i}$ parameters, listed in the fourth column of Table II. It can be seen that it dominates the uncertainty on $L_{i}$. For illustration, the area between the dotted lines in Fig. 3 corresponds to a Gaussian sampling of the chiral parameters with the two sources of error added in quadrature.

By comparing the $L_{i}^{r}$ from the IAM fit in Table II with those of previous ChPT determinations (in Table I), we see that there is perfect agreement between them. This comparison of the complete IAM fit parameters is possible only now that we have the full $O\left(p^{4}\right)$ amplitudes, given in Appendix $\mathrm{B}$, which are regularized and renormalized following the same scheme as in standard ChPT. In particular, the agreement in the value of $L_{7}$ indicates that we are including the effects of the $\eta^{\prime}$ consistently at lowest order.

The threshold parameters (scattering lengths and slope pa-

TABLE III. Scattering lengths $a_{I J}$ and slope parameters $b_{I J}$ for different meson-meson scattering channels. The experimental data come from $[10,55]$, the one-loop results from $[5,8,10]$, and those at two loops from [42]. We are using the definitions and conventions given in those references. Let us remark that our one-loop IAM results are closer to those of two-loop ChPT, although the IAM depends on many fewer parameters than the $O\left(p^{6}\right) \mathrm{ChPT}$.

\begin{tabular}{ccccc}
\hline \hline & Experiment & IAM fit & $\begin{array}{c}\text { ChPT } \\
O\left(p^{4}\right)\end{array}$ & $\begin{array}{c}\text { ChPT } \\
O\left(p^{6}\right)\end{array}$ \\
\hline$a_{00}$ & $0.26 \pm 0.05$ & $0.231_{-0.006}^{+0.003}$ & 0.20 & $0.219 \pm 0.005$ \\
$b_{00}$ & $0.25 \pm 0.03$ & $0.30 \pm 0.01$ & 0.26 & $0.279 \pm 0.011$ \\
$a_{20}$ & $-0.028 \pm 0.012$ & $-0.0411_{-0.001}^{+0.0009}$ & -0.042 & $-0.042 \pm 0.01$ \\
$b_{20}$ & $-0.082 \pm 0.008$ & $-0.074 \pm 0.001$ & -0.070 & $-0.0756 \pm 0.0021$ \\
$a_{11}$ & $0.038 \pm 0.002$ & $0.0377 \pm 0.0007$ & 0.037 & $0.0378 \pm 0.0021$ \\
$a_{1 / 20}$ & 0.13 to 0.24 & $0.11_{-0.09}^{+0.06}$ & 0.17 & \\
$a_{3 / 20}$ & -0.13 to -0.05 & $-0.049_{-0.003}^{+0.002}$ & -0.5 & \\
$a_{1 / 21}$ & 0.017 to 0.018 & $0.016 \pm 0.002$ & 0.014 & \\
$a_{10}$ & & $0.15_{-0.11}^{+0.07}$ & 0.0072 & \\
\hline \hline
\end{tabular}


rameters) obtained with the IAM are given in Table III for the low energy constants in the second column in Table II. The errors in Table III are obtained by a Gaussian sampling of the above low energy constants. Note that the experimental values of the threshold parameters have not been used as input in the fit, and the numbers we give are therefore predictions of the IAM. As we have anticipated before and Table III shows clearly, we are able to reproduce the low energy behavior with great accuracy. Let us then comment, for each different channel, on the results of the IAM fit.

\section{Channel $(I, J)=(1,1)$}

The most striking feature of this channel is the $\rho(770)$ resonance, which, as can be seen in Fig. 3(a), can be fitted with great precision. This had already been achieved at $O\left(p^{4}\right)$ with both the single [8] and the coupled [11] channel formalisms. However, it is now achieved in a simultaneous fit with all the other channels, but since we are using the complete $O\left(p^{4}\right)$ expressions we have a good description of the high energy data without spoiling the scattering lengths listed in Table III.

This channel depends very strongly on $2 L_{1}^{r}+L_{3}-L_{2}^{r}$, and this combination can thus be fitted with great accuracy. The mass and width from a clear Breit-Wigner resonance can be obtained from the phase shift by means of

$$
\delta_{I J}\left(M_{R}\right)=90^{\circ}, \quad \Gamma_{R}=\frac{1}{M_{R}}\left(\frac{d \delta_{I J}}{d s}\right)_{s=M_{R}^{2}}^{-1} .
$$

For the $(1,1)$ case we obtain $M_{\rho}=775.7_{-3.3}^{+4.3} \mathrm{MeV}$, and $\Gamma_{\rho}$ $=135.5_{-9.0}^{+8.0} \mathrm{MeV}$, in perfect agreement with the values given by the PDG [45]. The errors correspond to a Gaussian sampling with the central values quoted in the second column of Table II and the MINUIT errors of the fit.

Finally, and just for illustration, the inelasticity prediction from the IAM is shown in Fig. 3(d). Note that the data values are so small and the claimed precision is so tiny that any other effect not considered in this work (like the $4 \pi$ intermediate state) would yield a contribution beyond the precision we can expect to reach with the IAM. That is why these data have been excluded from the fit.

\section{Channel $(I, J)=(0,0)$}

There are three independent observables in this channel with data. Concerning the $\pi \pi$ scattering phase shift, plotted in Fig. 3(b), we can reproduce two resonant structures. First, there is the $\sigma$ [or $\left.f_{0}(400-1200)\right]$, which corresponds to a broad bump in the phase shift that gets as high as $50^{\circ}$ not very far from threshold. This is not a narrow Breit-Wigner resonance. Indeed, it was shown in the IAM with just one channel [8] that it is possible to find an associated pole in the second Riemann sheet, quite far from the real axis. Second, we can nicely reproduce the shape of the $f_{0}(980)$ which corresponds to a narrow Breit-Wigner resonance although over a background phase provided by the $\sigma$, so that its mass and width cannot be read directly from Eq. (34). Once more, it can be seen that the scattering lengths can also be reproduced simultaneously with the high energy data.

The next observable is the $\pi \pi \rightarrow K \bar{K}$ phase shift, Fig. 3(c), which can also be fitted neatly. Since we have included the $\eta \eta$ intermediate state, the fit is somewhat better than with just two channels above the two- $\eta$ threshold, as was suggested in [11], but not as much as expected (this could be due to our crude treatment of $\eta$ - $\eta^{\prime}$ mixing, which we noted at the end of Sec. II).

Finally, in Fig. 3(d), we show the inelasticity in the $(0,0)$ channel. These are the most controversial sets of data, since there is strong disagreement between several experiments (up to a factor of 2 in the overall normalization), as we mentioned when commenting on the data for this observable.

$$
\text { Channel }(I, J)=(2,0)
$$

We have plotted the results in Fig. 3(e). Since only the $\pi \pi$ state can have these quantum numbers, we are simply reproducing the single channel IAM formalism, which already gave a very good description of this nonresonant channel [9]. Nevertheless, let us remark that it is now fitted simultaneously with all the other channels, and the value of the scattering length obtained from our fit is compatible with the experimental result and standard ChPT (see Table III).

In addition, once we have a description of this and the $(0$, $0)$ channel, we can obtain the phase of the $\epsilon^{\prime}$ parameter which measures direct $C P$ violation in $K \rightarrow \pi \pi$ decays [48]. It is defined, in degrees, as follows:

$$
\phi\left(\epsilon^{\prime}\right)=90^{\circ}-\left(\delta_{00}-\delta_{20}\right)_{s=M_{K}^{2}} .
$$

Our result is $\phi\left(\epsilon^{\prime}\right)=38 \pm 0.3$, where the error is obtained from a Gaussian sampling of the parameters listed in column 2 of Table II with the MINUIT errors in the third column. This is in very good agreement with the experimentally observed value of $\phi\left(\epsilon^{\prime}\right)=43.5 \pm 7$. Standard ChPT [49] predicts 45 \pm 6 .

\section{Low energy $K_{l 4}$ decay data}

There is no real improvement in the description of these low energy data in Fig. 3(f) compared to ChPT, since standard ChPT works very well at these energies. However, these very precise data at such low energies ensure that the parameters of our fit cannot be too different from those of standard ChPT. In addition, they are extremely important in the determination of the scattering lengths, in particular, of the controversial $a_{00}$.

\section{Channel $(I, J)=(1 / 2,1)$}

As happened in the $(1,1)$ channel with the $\rho$, this channel is dominated by the $K^{*}(892)$. This is a distinct Breit-Wigner resonance that can be fitted very accurately with the IAM (see Fig. 3(g)). From Eq. (34) we find $M_{K^{*}}=889 \pm 5 \mathrm{MeV}$ and $\Gamma_{K^{*}}=46 \pm 13 \mathrm{MeV}$, in fairly good agreement with the PDG [45]. The errors were obtained in the same way as for the $\rho$ resonance in the $(1,1)$ channel. 


\section{Channel $(I, J)=(1 / 2,0)$}

Because of the wide dispersion of experimental results, our fit yields a wide error band for this channel, as can be seen in Fig. 3(h). Nevertheless, as happened in the $(0,0)$ channel, the phase shift is of the order of $50^{\circ}$ not far for threshold, due to a wide bump similar to the $\sigma$ in that channel. Here, this broad structure has been identified by different experimental and theoretical analyses $[50,9,51,46]$ as the $\kappa$ although there is still a controversy about its existence and origin [52], as also happened with the $\sigma$. It is very similar to the $\sigma$, and hence it cannot be interpreted as a Breit-Wigner narrow resonance.

We also give in Table III the value for the scattering length of this channel, in good agreement with the experimental data, which nevertheless are not very well known.

\section{Channel $(I, J)=(3 / 2,0)$}

Since only $\pi K$ can have these quantum numbers, this is once more the IAM with a single channel, which already provided a very good description [8]. We show in Fig. 3i the results of the global fit for this channel, as well as the corresponding scattering length in Table III.

\section{Channel $(I, J)=(1,0)$}

In our global fit, the data in this channel (see Fig. 3j) are reproduced using Eq. (33). The shape of the $a_{0}(980)$ is neatly reproduced in the mass distribution. In order to compare the value of the normalization constant $c$ with experiment, we also show in Fig. 4 the result of applying the IAM with the parameters obtained from our fit to the experimental data obtained from $K^{-} p \rightarrow \Sigma^{+}(1385) \pi \eta$ and $K^{-} p$ $\rightarrow \Sigma^{+}(1385) K \bar{K}$ [53]. These data have not been included in our fit since they do not have error bars, but it can be seen that the IAM provides a good description. Once again we are using a formula like Eq. (33), but with a constant different from that for Fig. $3 \mathrm{j}$ and no background. Our result is $c$ $=63 \pm 15 \mu \mathrm{b} / \mathrm{GeV}$, to be compared with the values quoted in [53] where $c$ was taken from 73 to $165 \mu \mathrm{b} / \mathrm{GeV}$.

$$
\text { Channel }(I, J)=(0,1)
$$

Finally, we show in Fig. 5 the results for the modulus of the amplitude in the $(0,1)$ channel. In this case, there is only one meson-meson scattering channel, namely, $K \bar{K} \rightarrow K \bar{K}$. Therefore, we can apply only the single channel IAM, and in so doing we find a pole at approximately $935 \mathrm{MeV}$ on the real axis. The width of this resonance is zero, since within our approach it can couple only to $K \bar{K}$ and its mass is below the two-kaon threshold. One is tempted to identify this resonance with the $\phi(1020)$ meson, but in fact it can only be related to its octet part $\omega_{8}$. The reason is that the singlet part $\omega_{1}$ is $\mathrm{SU}(3)$ symmetric and it does not couple to two mesons since their spatial function has to be antisymmetric. Consequently we can associate the resonance obtained with the IAM only with the octet $\omega_{8}[9,54]$. The position of the pole seems consistent with an intermediate mass between the $\phi(1020)$ and the $\omega(770)$. This state had also been found

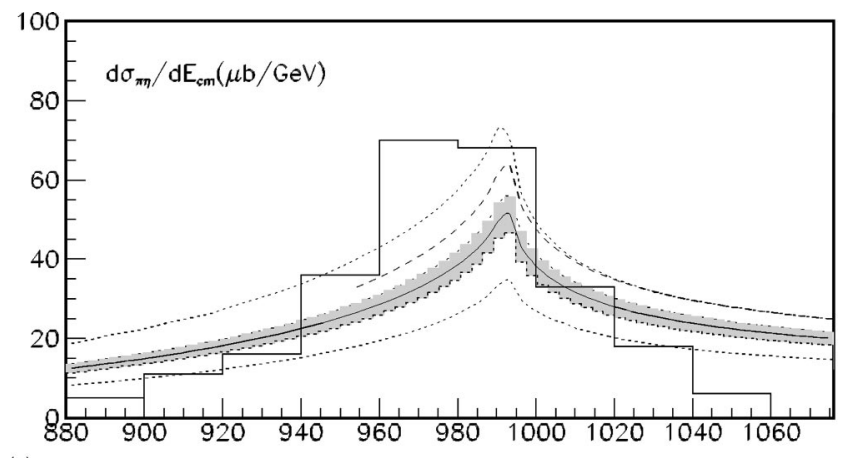

(a)

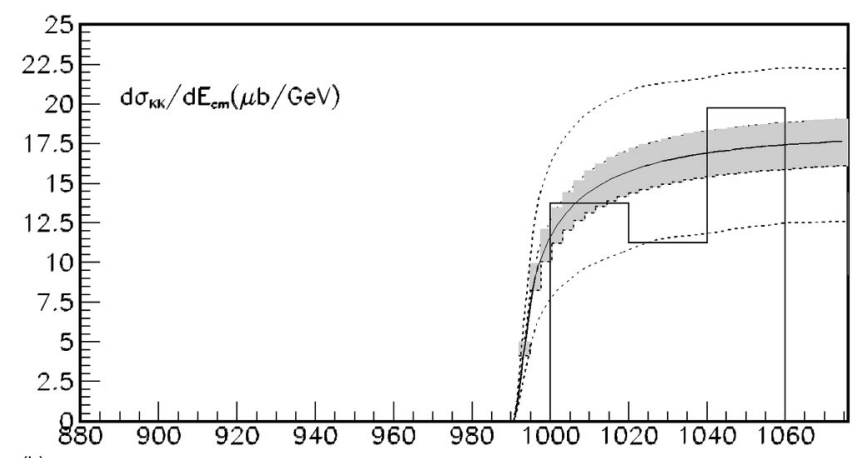

(b)

FIG. 4. We show the effective mass distributions of the two mesons in the final state of $K^{-} p \rightarrow \Sigma^{+}(1385) \pi \eta$ (top) and $K^{-} p$ $\rightarrow \Sigma^{+}(1385) K \bar{K}$ (bottom); the data come from [53]. The curves and bands are as in Fig. 3.

when using the IAM with incomplete chiral amplitudes [9], and it was used later to study the $\phi \rightarrow \pi \pi$ decay within a chiral unitary approach [54]. The fact that we find it here again confirms that it is not an artifact of the approximations used in [9]. In addition, although the amplitudes used here are complete up to $O\left(p^{4}\right)$ and the fit is rather different, it appears almost at the same place, which supports the soundness of the results in [9].

Finally, we have also added in Fig. 3 a dashed line that corresponds to the result with the central values of the parameters in the second column of Table II but where we have used the one-channel IAM at energies where there is only one state available, the two-channel IAM when there are

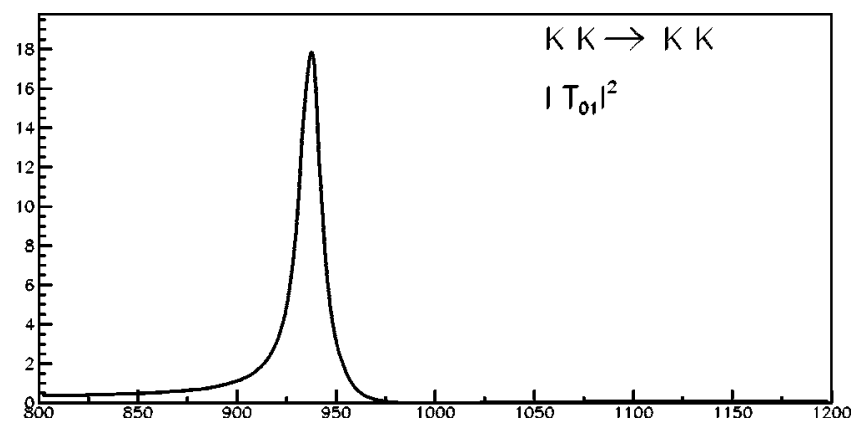

FIG. 5. We show the modulus of the $(I, J)=(0,1) \quad K \bar{K} \rightarrow K \bar{K}$ amplitude. The pole around 935 can be identified with the octet $\omega_{8}$ (see text for details). Although that cannot be shown in a plot, the modulus of the amplitude actually becomes infinite. 
two, etc. As we commented at the end of Sec. IV, this approach ensures exact unitarity at all energies, but we can see that it generates a discontinuity at each threshold. The results are compatible within the wider error bands with the previous IAM fit (the space between the dotted lines). This was expected since, as we have already commented, the difference between the two approaches is of the order of a few percent, which is also the order of magnitude of the systematic error added to the data for the fit. Of course, it is possible to obtain a fit with this method also, as done in [8], and the resulting parameters are still compatible with those listed in Table III.

\section{CONCLUSIONS}

In this work we have completed the calculation of the lightest octet meson-meson scattering amplitudes within chiral perturbation theory at one loop. We have calculated three new amplitudes $\eta \eta \rightarrow \eta \eta, K \eta \rightarrow K \eta$, and $K \eta \rightarrow K \pi$, but we have also recalculated the other five independent amplitudes, checking and revising previous results. The full expressions are given in Appendix B in a unified notation, using dimensional regularization and the $\overline{\mathrm{MS}}-1$ renormalization scheme, which is the usual one within ChPT. All the mesonmeson scattering partial waves below $1200 \mathrm{MeV}$, with definite isospin $I$ and angular momentum $J$, can be expressed in terms of these eight amplitudes.

Since ChPT is a low energy theory, the one-loop amplitudes have to be unitarized in order to reach energies as high as $1200 \mathrm{MeV}$ (and, in particular, the two-kaon threshold). For that purpose we have applied the coupled channel inverse amplitude method, which ensures unitarity for coupled channels and is also able to generate resonances and their associated poles, without introducing any additional parameter. In addition, it respects the chiral expansion at low energies, in our case up to $O\left(p^{4}\right)$. Thus, we have shown that it is possible to describe simultaneously the data on the $(I, J)$ $=(0,0),(1,1),(2,0),(1,0),(1 / 2,0),(1 / 2,1)$, and $(3 / 2,0)$ meson-meson channels below $1200 \mathrm{MeV}$, which correspond to 20 different reactions. We also describe seven resonant shapes, namely, the $\sigma, \rho(770), K^{*}(892), \quad \kappa, f_{0}(980)$, $a_{0}(980)$, and the octet $\omega_{8}$.

This description is achieved with values for the low energy constants that are perfectly compatible with previous determinations obtained using standard ChPT and low energy data. This comparison is possible only since we now have the complete $O\left(p^{4}\right)$ expression for all the amplitudes in the standard ChPT scheme. Indeed, with the present determinations of standard ChPT, we can already find the resonance shapes and we obtain the most distinct features of each channel, although with large uncertainties due to the present knowledge of the chiral parameters.

Nevertheless, we have performed a fit of our unitarized amplitudes to the meson-meson data and we have obtained a very accurate description not only of the resonance region, but also of the low energy data, and in particular of the scattering lengths. We have also paid particular attention to the uncertainties and errors in our description, which have been estimated with Monte Carlo samplings of the fitted chi- ral parameters within their resulting error bars.

Summarizing, we have extended and completed previous analyses using these techniques in the meson sector so that we believe that our present work will be useful for further phenomenological applications.

\section{ACKNOWLEDGMENTS}

We are very grateful to J. A. Oller for his comments, clarifications, and discussions about almost every issue addressed in this paper. We also thank J. Nieves and E. RuizArriola for providing and explaining to us the Monte Carlo code used to generate the error bands. In addition, we have profited from interesting discussions with A. Dobado and E. Oset. We acknowledge partial support from the Spanish CICYT projects FPA2000-0956, PB98-0782, and BFM20001326.

\section{APPENDIX A: USEFUL FORMULAS}

Here we will give the main results and definitions of the different functions coming from the one-loop ChPT calculation. We are following the notation and conventions of [3].

When calculating the ChPT amplitudes, the typical loop integrals that appear are, on the one hand, the tadpole integral, i.e., the Feynman boson propagator evaluated at $x=0$ :

$$
\int \frac{d^{d} q}{(2 \pi)^{d}} \frac{i}{q^{2}-M_{i}^{2}}=2 M_{i}^{2} \lambda+\frac{M_{i}^{2}}{16 \pi^{2}} \log \frac{M_{i}^{2}}{\mu^{2}}
$$

where $\mu$ is the renormalization scale, $i=\pi, K, \eta$, and we have extracted its divergent part for $d \rightarrow 4$, with $\lambda$ given in Eq. (5). On the other hand, the integral coming from diagrams (a), (b), and (c) in Fig. 1 is:

$$
J_{P Q}\left(p^{2}\right)=-i \int \frac{d^{d} q}{(2 \pi)^{d}} \frac{1}{\left[q^{2}-M_{P}^{2}\right]\left[(q-p)^{2}-M_{Q}^{2}\right]}
$$

where $P, Q=\pi, K, \eta$ and whose divergent contribution in dimensional regularization can be separated as

$$
J_{P Q}(s)=J_{P Q}(0)+\bar{J}_{P Q}(s)+O(d-4),
$$

where

$$
\begin{aligned}
J_{P Q}(0)= & -2 \lambda-\frac{1}{16 \pi^{2}} \frac{1}{\Delta}\left[M_{P}^{2} \log \frac{M_{P}^{2}}{\mu^{2}}-M_{Q}^{2} \log \frac{M_{Q}^{2}}{\mu^{2}}\right], \\
\bar{J}_{P Q}(S)= & \frac{1}{32 \pi^{2}}\left[2+\left(\frac{\Delta}{s}-\frac{\Sigma}{\Delta}\right) \log \frac{M_{Q}^{2}}{M_{P}^{2}}\right. \\
& \left.-\frac{\nu(s)}{s} \log \frac{[s+\nu(s)]^{2}-\Delta^{2}}{[s-\nu(s)]^{2}-\Delta^{2}}\right]
\end{aligned}
$$

and

$$
\begin{aligned}
& \Delta=M_{P}^{2}-M_{Q}^{2}, \\
& \Sigma=M_{P}^{2}+M_{Q}^{2},
\end{aligned}
$$




$$
\nu^{2}(s)=\left[s-\left(M_{P}+M_{Q}\right)^{2}\right]\left[s-\left(M_{P}-M_{Q}\right)^{2}\right] .
$$

For the case of a single mass $M_{P}=M_{Q}$, the above integrals read

$$
\begin{aligned}
& J_{P P}(s)=-2 \lambda-\frac{1}{16 \pi^{2}}\left(1+\log \frac{M_{P}^{2}}{\mu^{2}}\right)+\bar{J}_{P P}(s), \\
& \bar{J}_{P P}(s)=\frac{1}{16 \pi^{2}}\left[2+\sigma(s) \log \frac{\sigma(s)-1}{\sigma(s)+1}\right],
\end{aligned}
$$

with

$$
\sigma(s)=\left(1-4 M_{P}^{2} / s\right)^{1 / 2}
$$

Note that the above integrals have the correct unitarity structure in the right cut, which extends on the real axis from $s=\left(M_{P}+M_{Q}\right)^{2}$ to infinity. In fact, all the integrals appearing to one loop in ChPT can be expressed in terms of the tadpole and $\bar{J}$ integrals above [3]. However, it is customary to express the results also in terms of

$$
\overline{\bar{J}}_{P Q}(s) \equiv \bar{J}(s)-s \bar{J}^{\prime}(0),
$$

where from Eq. (A4) one has

$$
\bar{J}^{\prime}(0)=\frac{1}{32 \pi^{2}}\left[\frac{\Sigma}{\Delta^{2}}+2 \frac{M_{P}^{2} M_{Q}^{2}}{\Delta^{3}} \log \frac{M_{Q}^{2}}{M_{P}^{2}}\right] .
$$

From the above definitions it is easy to check that the functions $\bar{J}(s) / s$ and $\overline{\bar{J}}(s) / s^{2}$ have well-defined limits as $s$ $\rightarrow 0$.

\section{APPENDIX B: ONE LOOP AMPLITUDES FROM CHPT}

Here we list the expressions for the eight independent meson-meson scattering amplitudes to one loop in ChPT. We have carefully checked the scale independence and perturbative exact unitary (see Sec. III). Note that we have used Eq. (9) to write all the $f_{K}$ and $f_{\eta}$ in terms of $f_{\pi}, L_{4}^{r}$, and $L_{5}^{r}$, in order to ensure "exact" perturbative unitarity, Eq. (27). Let us first give the three amplitudes that had never before appeared in the literature in any form.

For $\eta \eta \rightarrow \eta \eta$,

$$
\begin{aligned}
T(s, t, u)= & \frac{16 M_{K}^{2}-7 M_{\pi}^{2}}{9 f_{\pi}^{2}}+\frac{\mu_{\pi}}{9 f_{\pi}^{2}}\left\{7 M_{\pi}^{2}-48 M_{\eta}^{2}\right\}-\frac{\mu_{K}}{18 f_{\pi}^{2} M_{K}^{2}}\left\{81\left[t^{2}-s u-4 t M_{\eta}^{2}\right]+14 M_{\pi}^{4}-48 M_{\pi}^{2} M_{\eta}^{2}+378 M_{\eta}^{4}\right\} \\
& -\frac{\mu_{\eta}}{3 f_{\pi}^{2} M_{\eta}^{2}}\left\{M_{\pi}^{4}-8 M_{\pi}^{2} M_{\eta}^{2}+24 M_{\eta}^{4}\right\}+\frac{4}{f_{\pi}^{4}}\left(2 L_{1}^{r}+2 L_{2}^{r}+L_{3}\right)\left\{s^{2}+t^{2}+u^{2}-4 M_{\eta}^{4}\right\}-\frac{8}{3 f_{\pi}^{4}}\left\{12 M_{\eta}^{4} L_{4}^{r}+\left(3 M_{\pi}^{4}\right.\right. \\
& \left.\left.-10 M_{\pi}^{2} M_{\eta}^{2}+13 M_{\eta}^{4}\right) L_{5}^{r}-36 M_{\eta}^{4} L_{6}^{r}-24\left(M_{\pi}^{4}-3 M_{\pi}^{2} M_{\eta}^{2}+2 M_{\eta}^{4}\right) L_{7}-6 L_{8}^{r}\left(2 M_{\pi}^{4}-6 M_{\pi}^{2} M_{\eta}^{2}+7 M_{\eta}^{4}\right)\right\} \\
& -\frac{1}{192 \pi^{2} f_{\pi}^{4}}\left\{27\left(t^{2}-s u-4 t M_{\eta}^{2}\right)+16\left(23 M_{\eta}^{4}-22 M_{K}^{2} M_{\eta}^{2}+10 M_{K}^{4}\right)\right\}+\frac{1}{6 f_{\pi}^{4}}\left\{\frac{1}{27}\left(16 M_{K}^{2}-7 M_{\pi}^{2}\right)^{2} \bar{J}_{\eta \eta}(s)\right. \\
& \left.+M_{\pi}^{4} \bar{J}_{\pi \pi}(s)+\frac{1}{12}\left(9 s-2 M_{\pi}^{2}-6 M_{\eta}^{2}\right)^{2} \bar{J}_{K K}(s)+[s \leftrightarrow t]+[s \leftrightarrow u]\right\} .
\end{aligned}
$$

For $\bar{K}^{0} \eta \rightarrow \bar{K}^{0} \eta$,

$$
\begin{aligned}
T(s, t, u)= & \frac{9 t-6 M_{\eta}^{2}-2 M_{\pi}^{2}}{12 f_{\pi}^{2}}-\frac{2 L_{5}^{r}}{3 f_{\pi}^{4}}\left[3 M_{\pi}^{4}+12 M_{\eta}^{4}+M_{\pi}^{2}\left(5 M_{\eta}^{2}-9 t\right)\right]+\frac{1}{3 f_{\pi}^{4}}\left\{2\left(12 L_{1}^{r}+5 L_{3}^{r}\right)\left(2 M_{K}^{2}-t\right)\left(2 M_{\eta}^{2}-t\right)+\left(12 L_{2}^{r}+L_{3}\right)\right. \\
& \left.\times\left[\left(s-M_{K}^{2}-M_{\eta}^{2}\right)^{2}+\left(u-M_{K}^{2}-M_{\eta}^{2}\right)^{2}\right]\right\}+\frac{4}{f_{\pi}^{4}}\left\{8\left(L_{6}^{r}-L_{4}^{r}\right) M_{K}^{2} M_{\eta}^{2}+2 L_{7}\left(M_{\pi}^{4}-4 M_{\pi}^{2} M_{\eta}^{2}+3 M_{\eta}^{4}\right)\right. \\
& \left.+L_{8}^{r}\left(M_{\pi}^{4}-3 M_{\pi}^{2} M_{\eta}^{2}+6 M_{\eta}^{4}\right)+2 L_{4}^{r} t\left(M_{\eta}^{2}+M_{K}^{2}\right)\right\}-\frac{\mu_{\pi}}{48 f_{\pi}^{2}\left(M_{K}^{2}-M_{\eta}^{2}\right)}\left\{2 M_{K}^{2}\left[26 M_{\eta}^{2}+69 t\right]-84 M_{K}^{4}\right. \\
& \left.+3\left[16 M_{\eta}^{4}-50 t M_{\eta}^{2}+(s-u)^{2}\right]\right\}-\frac{\mu_{K}}{72 f_{\pi}^{2} M_{K}^{2}\left(M_{K}^{2}-M_{\eta}^{2}\right)}\left\{92 M_{K}^{6}-81 M_{\eta}^{2} t^{2}-60 M_{K}^{4}\left[3 t+M_{\eta}^{2}\right]\right. \\
& \left.+18 M_{K}^{2}\left(5 t^{2}-2 s u+6 t M_{\eta}^{2}+8 M_{\eta}^{4}\right)\right\}+\frac{\mu_{\eta}}{144 f_{\pi}^{2} M_{\eta}^{2}\left(M_{K}^{2}-M_{\eta}^{2}\right)}\left\{144 t M_{K}^{4}-128 M_{K}^{6}+\left[27(s-u)^{2}-486 t M_{K}^{2}\right.\right. \\
& \left.\left.+428 M_{K}^{4}\right] M_{\eta}^{2}+2\left[153 t-166 M_{K}^{2}\right] M_{\eta}^{4}+144 M_{\eta}^{6}\right\}+\frac{1}{2304 f_{\pi}^{4} \pi^{2}}\left\{116 M_{K}^{4}+M_{K}^{2}\left[184 M_{\eta}^{2}-153 t\right]\right.
\end{aligned}
$$




$$
\begin{aligned}
& \left.-9\left[10 t^{2}+2 s u-3 t M_{\eta}^{2}+4 M_{\eta}^{4}\right]\right\}+\frac{t \bar{J}_{K K}(t)\left(9 t-2 M_{\pi}^{2}-6 M_{\eta}^{2}\right)}{16 f_{\pi}^{4}}+\frac{\bar{J}_{\eta \eta}(t)\left(9 t-2 M_{\pi}^{2}-6 M_{\eta}^{2}\right)\left(16 M_{K}^{2}-7 M_{\pi}^{2}\right)}{216 f_{\pi}^{4}} \\
& +\frac{t \bar{J}_{\pi \pi}(t) M_{\pi}^{2}}{8 f_{\pi}^{4}}+\frac{1}{32 f_{\pi}^{4}}\left\{\frac { \overline { J } _ { K \eta } ( s ) } { 9 } \left[27 s(s-u)+189 M_{K}^{4}+8 M_{\pi}^{4}+54 u M_{\eta}^{2}+45 M_{\eta}^{4}+12 M_{\pi}^{2}\left(3 s-2 M_{\eta}^{2}\right)\right.\right. \\
& \left.-18 M_{K}^{2}\left(6 s-3 u+4 M_{\pi}^{2}+9 M_{\eta}^{2}\right)\right]+\frac{\bar{J}_{K \pi}(s)}{9}\left[27 s(s-u)+29 M_{K}^{4}+11 M_{\pi}^{4}+18 M_{\eta}^{4}+2 M_{K}^{2}\left(18 s+27 u-47 M_{\pi}^{2}\right.\right. \\
& \left.\left.-78 M_{\eta}^{2}\right)+6 M_{\pi}^{2}\left(9 u-6 s+8 M_{\eta}^{2}\right)\right]-\frac{\bar{J}_{K \pi}(s)}{s}\left\{M_{K}^{4}\left(3 u+14 M_{\pi}^{2}-8 M_{\eta}^{2}\right)+2 M_{K}^{6}-2 M_{K}^{2} M_{\pi}^{2}\left(3 u+5 M_{\pi}^{2}+4 M_{\eta}^{2}\right)\right. \\
& \left.+M_{\pi}^{2}\left[6 M_{\eta}^{4}+M_{\pi}^{2}\left(3 u+4 M_{\eta}^{2}\right)\right]\right\}+\frac{\bar{J}_{K \eta}(s)}{s}\left(M_{K}^{2}-M_{\eta}^{2}\right)^{2}\left(4 M_{\pi}^{2}-18 M_{K}^{2}-6 M_{\eta}^{2}-3 u\right)+6\left(M_{K}^{2}-M_{\eta}^{2}\right)^{2} \\
& \left.\times \frac{\overline{\bar{J}}_{K \pi}(s)\left(M_{K}^{2}-M_{\pi}^{2}\right)^{2}+\overline{\bar{J}}_{K \eta}(s)\left(M_{K}^{2}-M_{\eta}^{2}\right)^{2}}{s^{2}}+[s \leftrightarrow u]\right\} .
\end{aligned}
$$

For $\bar{K}^{0} \eta \rightarrow \bar{K}^{0} \pi^{0}$,

$$
\begin{aligned}
T(s, t, u)= & \frac{8 M_{K}^{2}+3 M_{\eta}^{2}+M_{\pi}^{2}-9 t}{12 \sqrt{3} f_{\pi}^{2}}+\frac{\mu_{\pi}}{48 \sqrt{3} f_{\pi}^{2}\left(M_{K}^{2}-M_{\pi}^{2}\right)}\left\{27 s^{2}+18 s u+27 u^{2}+174 t M_{K}^{2}-292 M_{K}^{4}+12\left(5 M_{K}^{2}-6 t\right) M_{\pi}^{2}\right. \\
& \left.-32 M_{\pi}^{4}\right\}-\frac{\mu_{K}}{24 \sqrt{3} f_{\pi}^{2} M_{K}^{2}\left(M_{K}^{2}-M_{\pi}^{2}\right)}\left\{9 t^{2} M_{\pi}^{2}+24 M_{K}^{6}+4 M_{K}^{4}\left(17 M_{\pi}^{2}-15 t\right)+2 M_{K}^{2}\left[9(s-u)^{2}+6 t M_{\pi}^{2}-22 M_{\pi}^{4}\right]\right\} \\
& -\frac{\mu_{\eta}}{16 \sqrt{3} f_{\pi}^{2}\left(M_{K}^{2}-M_{\eta}^{2}\right)}\left\{3(s-u)^{2}+2\left(3 t-14 M_{K}^{2}+10 M_{\eta}^{2}\right)\left(M_{k}^{2}-2 M_{\eta}^{2}\right)\right\}+\frac{1}{256 \sqrt{3} \pi^{2} f_{\pi}^{4}}\left\{2(2 s+u)(s+2 u)-192 M_{K}^{4}\right. \\
& \left.-23 t M_{\eta}^{2}-16 M_{\eta}^{4}+5 M_{K}^{2}\left(13 t+24 M_{\eta}^{2}\right)\right\}-\frac{L_{3}}{\sqrt{3} f_{\pi}^{4}}\left\{s^{2}+4 s u+u^{2}-30 M_{K}^{4}-2 t M_{\eta}^{2}+2 M_{\eta}^{4}+6 M_{K}^{2}\left(t+2 M_{\eta}^{2}\right)\right\} \\
& +\frac{1}{\sqrt{3} f_{\pi}^{4}}\left\{3 M_{\pi}^{4}\left[L_{5}^{r}-2\left(2 L_{7}+L_{8}^{r}\right)\right]+M_{\eta}^{4}\left[6\left(2 L_{7}+L_{8}^{r}\right)-L_{5}^{r}\right]-6 L_{5}^{r} M_{\pi}^{2}\left(t-M_{\eta}^{2}\right)\right\}-\frac{9 t-8 M_{K}^{2}-M_{\pi}^{2}-3 M_{\eta}^{2}}{144 \sqrt{3} f_{\pi}^{4}} \\
& \left.+43 M_{\pi}^{2} \bar{J}_{\pi \eta}(t)\right]+\frac{1}{288 \sqrt{3} f_{\pi}^{4}}\left\{\overline { J } _ { K \eta } ( s ) \left[27 s(u-s)-45 M_{K}^{4}+14 M_{\pi}^{4}-6 M_{\eta}^{2}\left(9 u+7 M_{\pi}^{2}\right)-9 M_{\eta}^{4}+M_{K}^{2}(36 s-54 u\right.\right. \\
& \left.\left.+22 M_{\pi}^{2}+156 M_{\eta}^{2}\right)\right]+3 \bar{J}_{K \pi}(s)\left[29 M_{K}^{4}+7 M_{\pi}^{4}+3 s\left(9 s+3 u-4 M_{\eta}^{2}\right)-2 M_{K}^{2}\left(16 s+9 u-18 M_{\pi}^{2}+3 M_{\eta}^{2}\right)\right. \\
& \left.-M_{\pi}^{2}\left(40 s+18 u-30 M_{\eta}^{2}\right)\right]+9 \frac{\bar{J}_{K \eta}(s)}{s}\left(M_{K}^{2}-M_{\eta}^{2}\right)\left[10 M_{K}^{4}+2 M_{\pi}^{4}-M_{\eta}^{2}\left(3 u+8 M_{\pi}^{2}\right)+M_{K}^{2}\left(3 u-12 M_{\pi}^{2}+8 M_{\eta}^{2}\right)\right] \\
& +9 \frac{\bar{J}_{K \pi}(s)}{s}\left(M_{K}^{2}-M_{\pi}^{2}\right)^{2}\left(3 u-2 M_{K}^{2}+2 M_{\pi}^{2}\right)-\frac{54 \bar{J}_{K \eta}(s)}{s^{2}}\left(M_{K}^{2}-M_{\pi}^{2}\right)\left(M_{K}^{2}-M_{\eta}^{2}\right)^{3}-\frac{54 \bar{J}_{K \pi}(s)}{s^{2}}\left(M_{K}^{2}-M_{\pi}^{2}\right)^{3} \\
& \left.\times\left(M_{K}^{2}-M_{\eta}^{2}\right)+[s \leftrightarrow u]\right\} \cdot . \\
& (\mathrm{B} 3) \\
&
\end{aligned}
$$

Apart from the above three amplitudes, we have recalculated the other independent five. The reason is threefold. First, we wanted them to satisfy exact perturbative unitarity to apply the simplest IAM formulas. This was not the case of all the calculations in the literature, even when considering the one-channel case. Second, there have been several unfortunate misprints and errata in the published formulas (including some errata made by one of us). Finally we would like to have a self-contained description of the one-loop calculation, together with all the resulting formulas. Nevertheless, when compared with previous analyses, our results are not exactly the same because we have chosen to express the amplitudes in terms of only 
one physical decay constant $f_{\pi}$, and we have used the Gell-Mann-Okubo relation only to simplify masses if it did not affect the exact perturbative unitarity relation. Apart from previous misprints, the differences are $O\left(p^{6}\right)$. The first amplitude to appear in the literature was $\pi^{+} \pi^{-} \rightarrow \pi^{0} \pi^{0}$, although in SU(2) [2]. However, we have been able to check also with the SU(3) calculation [10]. The result, following the notation in Appendix A, is

$$
\begin{aligned}
T(s, t, u)= & \frac{s-M_{\pi}^{2}}{f_{\pi}^{2}}-\frac{\mu_{\pi}}{3 f_{\pi}^{2} M_{\pi}^{2}}\left\{4 s^{2}-4 t u-4 s M_{\pi}^{2}+9 M_{\pi}^{4}\right\}-\frac{\mu_{K}}{6 f_{\pi}^{2} M_{K}^{2}}\left\{s^{2}-t u+2 s M_{\pi}^{2}\right\}-\frac{\mu_{\eta} M_{\pi}^{4}}{9 f_{\pi}^{2} M_{\eta}^{2}}+\frac{4}{f_{\pi}^{4}}\left\{\left(2 L_{1}^{r}+L_{3}\right)\left(s-2 M_{\pi}^{2}\right)^{2}\right. \\
& \left.+L_{2}^{r}\left[\left(t-2 M_{\pi}^{2}\right)^{2}+\left(u-2 M_{\pi}^{2}\right)^{2}\right]\right\}+\frac{8 M_{\pi}^{2}}{f_{\pi}^{4}}\left\{\left(2 L_{4}^{r}+L_{5}^{r}\right) s+2\left(2 L_{6}^{r}+L_{8}^{r}-2 L_{4}^{r}-L_{5}^{r}\right) M_{\pi}^{2}\right\}+\frac{1}{576 \pi^{2} f_{\pi}^{4}}\left\{30\left(M_{\pi}^{2}-s\right) s\right. \\
& \left.+21 t u-56 M_{\pi}^{4}\right\}+\frac{1}{2 f_{\pi}^{4}}\left\{\frac{s^{2} \bar{J}_{K K}(s)}{4}+\frac{M_{\pi}^{4} \bar{J}_{\eta \eta}(s)}{9}+\left(s^{2}-M_{\pi}^{4}\right) \bar{J}_{\pi \pi}(s)\right\}+\frac{1}{6 f_{\pi}^{4}}\left\{\frac{\left(t-4 M_{K}^{2}\right)\left(2 s+t-4 M_{\pi}^{2}\right) \bar{J}_{K K}(t)}{4}\right. \\
& \left.+\left[t(t-u)-2 M_{\pi}^{2}\left(t-2 u+M_{\pi}^{2}\right)\right] \bar{J}_{\pi \pi}(t)+[t \leftrightarrow u]\right\} .
\end{aligned}
$$

The $K^{+} \pi^{+} \rightarrow K^{+} \pi^{+}$one-loop calculation was first given in [10]. It was correct up to $O\left(p^{4}\right)$ but when expressed in terms of physical constants it did not satisfy exact perturbative unitarity. One of us gave an expression satisfying that relation [8], but there was also a typographical error in that reference. Our corrected result, expressed just in terms of $f_{\pi}$ is

$$
\begin{aligned}
& T^{3 / 2}(s, t, u)=\frac{M_{K}^{2}+M_{\pi}^{2}-s}{2 f_{\pi}^{2}}+\frac{2}{f_{\pi}^{4}}\left\{\left(4 L_{1}^{r}+L_{3}\right)\left(t-2 M_{K}^{2}\right)\left(t-2 M_{\pi}^{2}\right)+\left(2 L_{2}^{r}+L_{3}\right)\left(u-M_{K}^{2}-M_{\pi}^{2}\right)^{2}+2 L_{2}^{r}\left(s-M_{K}^{2}-M_{\pi}^{2}\right)^{2}\right. \\
& \left.+4 L_{4}^{r}\left[t M_{\pi}^{2}+M_{K}^{2}\left(t-4 M_{\pi}^{2}\right)\right]-2 L_{5}^{r} M_{\pi}^{2}\left(s+M_{K}^{2}-M_{\pi}^{2}\right)+8\left(2 L_{6}^{r}+L_{8}^{r}\right) M_{K}^{2} M_{\pi}^{2}\right\}+\frac{\mu_{\pi}}{24 f_{\pi}^{2} M_{\pi}^{2}\left(M_{\pi}^{2}-M_{K}^{2}\right)}\left\{2 M_{K}^{2}(7 s\right. \\
& \left.\left.+5 u-12 M_{K}^{2}\right)\left(2 M_{\pi}^{2}-t\right)-\left[26 s^{2}+21 s u+25 u^{2}-3 M_{K}^{2}\left(s+5 u+16 M_{K}^{2}\right)\right] M_{\pi}^{2}+\left(85 s+53 u-78 M_{K}^{2}\right) M_{\pi}^{4}-66 M_{\pi}^{6}\right\} \\
& +\frac{\mu_{K}}{12 f_{\pi}^{2} M_{K}^{2}\left(M_{\pi}^{2}-M_{K}^{2}\right)}\left\{42 M_{K}^{6}-M_{\pi}^{2}\left(5 s+4 u-9 M_{\pi}^{2}\right)\left(2 M_{K}^{2}-t\right)+4 M_{K}^{4}\left(12 M_{\pi}^{2}-13 s-8 u\right)+M_{K}^{2}\left[11 s^{2}+12 s u\right.\right. \\
& \left.\left.+7 u^{2}-3 M_{\pi}^{2}\left(s-u+8 M_{\pi}^{2}\right)\right]\right\}+\frac{\mu_{\eta}}{72 f_{\pi}^{2} M_{\eta}^{2}\left(M_{\eta}^{2}-M_{\pi}^{2}\right)}\left\{41 M_{\pi}^{6}-18(s+u) M_{\pi}^{4}+\left[36(s-u) u+9(s+5 u) M_{\pi}^{2}\right.\right. \\
& \left.\left.-59 M_{\pi}^{4}\right] M_{\eta}^{2}-3\left[9(5 s+u)-43 M_{\pi}^{2}\right] M_{\eta}^{4}+81 M_{\eta}^{6}\right\}+\frac{1}{1152 f_{\pi}^{4} \pi^{2}}\left\{3(s-10 t) t-6 s u-3 u^{2}-27 M_{K}^{4}+M_{K}^{2}(30 s-3 t\right. \\
& \left.\left.+21 u-34 M_{\pi}^{2}\right)-3 M_{\pi}^{2}\left(t-2 s-3 u+M_{\pi}^{2}\right)\right\}-\frac{\bar{J}_{K \pi}(u)}{16 f_{\pi}^{4}}\left\{(s-5 u) u+5 M_{K}^{4}-2(s-2 u) M_{\pi}^{2}+5 M_{\pi}^{4}-2 M_{K}^{2}(s-2 u\right. \\
& \left.\left.+5 M_{\pi}^{2}\right)\right\}+\frac{\bar{J}_{\pi \pi}(t)}{24 f_{\pi}^{4}}\left\{t\left(5 t-2 s+2 M_{K}^{2}\right)+\left(8 s+3 t-8 M_{K}^{2}\right) M_{\pi}^{2}-8 M_{\pi}^{4}\right\}-\frac{\bar{J}_{\eta \eta}(t) M_{\pi}^{2}}{72 f_{\pi}^{4}}\left[2 M_{\pi}^{2}+6 M_{\eta}^{2}-9 t\right] \\
& +\frac{\bar{J}_{K K}(t)}{24 f_{\pi}^{4}}\left\{M_{K}^{2}\left(4 s+3 t-4 M_{\pi}^{2}\right)+t\left(4 t-s+M_{\pi}^{2}\right)-4 M_{K}^{4}\right\}+\frac{\bar{J}_{K \eta}(u)}{432 f_{\pi}^{4}}\left\{2 M_{K}^{2}\left(27 s+18 u-74 M_{\pi}^{2}-51 M_{\eta}^{2}\right)+29 M_{K}^{4}\right. \\
& \left.+38 M_{\pi}^{4}-M_{\pi}^{2}\left(36 u-48 M_{\eta}^{2}\right)-9\left(3(s-u) u-6 s M_{\eta}^{2}+M_{\eta}^{4}\right)\right\}+\frac{\bar{J}_{K \pi}(s)}{4 f_{\pi}^{4}}\left(M_{K}^{2}+M_{\pi}^{2}-s\right)^{2}-\frac{\bar{J}_{K \eta}(u)}{48 f_{\pi}^{4} u}\left\{2 M_{K}^{6}\right. \\
& \left.+10 M_{\pi}^{4} M_{\eta}^{2}+3 s M_{\eta}^{4}+M_{K}^{4}\left(3 s-4 M_{\pi}^{2}+10 M_{\eta}^{2}\right)-2 M_{K}^{2}\left(2 M_{\pi}^{4}+\left(3 s+4 M_{\pi}^{2}\right) M_{\eta}^{2}+3 M_{\eta}^{4}\right)\right\}-\left(M_{K}^{2}\right. \\
& \left.-M_{\pi}^{2}\right)^{2} \frac{\bar{J}_{K \pi}(u)\left(s-2 M_{K}^{2}-2 M_{\pi}^{2}\right)}{16 f_{\pi}^{4} u}+\left(M_{K}^{2}-M_{\pi}^{2}\right)^{2} \frac{\left(M_{K}^{2}-M_{\eta}^{2}\right)^{2} \overline{\bar{J}}_{K \eta}(u)+\left(M_{K}^{2}-M_{\pi}^{2}\right)^{2} \overline{\bar{J}}_{K \pi}(u)}{8 f_{\pi}^{4} u^{2}} .
\end{aligned}
$$

The one-loop $\pi^{0} \eta \rightarrow \pi^{0} \eta$ amplitude was calculated in [10]. We give here the result expressed in terms of physical quantities: 


$$
\begin{aligned}
T(s, t, u)= & \frac{M_{\pi}^{2}}{3 f_{\pi}^{2}}-\frac{\left(13 M_{\pi}^{4}+6 t\left(M_{\pi}^{2}-M_{\eta}^{2}\right)-9 M_{\pi}^{2} M_{\eta}^{2}\right) \mu_{\pi}}{9 f_{\pi}^{2}\left(M_{\pi}^{2}-M_{\eta}^{2}\right)}+\frac{\left(M_{\pi}^{6}-M_{\pi}^{4} M_{\eta}^{2}+4 M_{\pi}^{2} M_{\eta}^{4}\right) \mu_{\eta}}{9 f_{\pi}^{2} M_{\eta}^{2}\left(M_{\pi}^{2}-M_{\eta}^{2}\right)}-\frac{\mu_{K}}{6 f_{\pi}^{2} M_{K}^{2}}\left\{20 M_{\pi}^{2}\left(t-3 M_{\eta}^{2}\right)-25 M_{\pi}^{4}\right. \\
& \left.+3\left[3\left(s^{2}+s u+u^{2}\right)+8 t M_{\eta}^{2}-9 M_{\eta}^{4}\right]\right\}+\frac{4}{f_{\pi}^{4}}\left\{2\left(L_{1}^{r}+L_{3} / 6\right)\left(t-2 M_{\pi}^{2}\right)\left(t-2 M_{\eta}^{2}\right)+\left(L_{2}^{r}+L_{3} / 3\right)\left[\left(s-M_{\pi}^{2}-M_{\eta}^{2}\right)^{2}\right.\right. \\
& \left.\left.+\left(u-M_{\pi}^{2}-M_{\eta}^{2}\right)^{2}\right]\right\}+\frac{8}{f_{\pi}^{4}}\left\{\left[t\left(M_{\pi}^{2}+M_{\eta}^{2}\right)-4 M_{\pi}^{2} M_{\eta}^{2}\right] L_{4}^{r}+2\left(2 L_{6}^{r}-L_{5}^{r} / 3\right) M_{\pi}^{2} M_{\eta}^{2}+4 L_{7} M_{\pi}^{2}\left(M_{\pi}^{2}-M_{\eta}^{2}\right)+2 L_{8}^{r} M_{\pi}^{4}\right\} \\
& +\frac{1}{576 f_{\pi}^{4} \pi^{2}}\left\{77 M_{\pi}^{4}+M_{\pi}^{2}\left(154 M_{\eta}^{2}-72 t\right)-9\left[3\left(s^{2}+s u+u^{2}\right)+8 t M_{\eta}^{2}-9 M_{\eta}^{4}\right]\right\}+\frac{1}{6 f_{\pi}^{4}}\left\{\frac{M_{\pi}^{2}}{9} \bar{J}_{\eta \eta}(t)\left(16 M_{K}^{2}-7 M_{\pi}^{2}\right)\right. \\
& \left.+\frac{t}{4} \bar{J}_{K K}(t)\left(9 t-2 M_{\pi}^{2}-6 M_{\eta}^{2}\right)+\bar{J}_{\pi \pi}(t) M_{\pi}^{2}\left(2 t-M_{\pi}^{2}\right)\right\}+\frac{1}{9 f_{\pi}^{4}}\left\{M_{\pi}^{4} \bar{J}_{\pi \eta}(s)+\frac{1}{24} \bar{J}_{K K}(s)\left(9 s-8 M_{K}^{2}-M_{\pi}^{2}-3 M_{\eta}^{2}\right)^{2}\right. \\
& +[s \leftrightarrow u]\} .
\end{aligned}
$$

Finally, the $K K$ scattering amplitudes were calculated in [11]. They were given in a rather different notation from the previous ones. Our result is, for $K^{+} K^{-} \rightarrow K^{+} K^{-}$,

$$
\begin{aligned}
T_{\mathrm{ch}}(s, t, u)= & \frac{2 M_{K}^{2}-u}{f_{\pi}^{2}}-\frac{\mu_{K}}{6 f_{\pi}^{2} M_{K}^{2}}\left[5\left(s^{2}+s t+t^{2}\right)+6 u^{2}-13 u M_{K}^{2}-8 M_{K}^{4}\right]+\frac{\mu_{\pi}}{2 f_{\pi}^{2}}\left\{5\left(u-2 M_{K}^{2}\right)\right. \\
& \left.-\frac{11 s^{2}+8 s t+11 t^{2}+8 u M_{K}^{2}-32 M_{K}^{4}}{24 M_{\pi}^{2}}+\frac{\left.9\left(s^{2}+t^{2}\right)+24 u M_{K}^{2}-64 M_{K}^{4}\right\}}{16\left(M_{K}^{2}-M_{\pi}^{2}\right)}\right\}+\frac{\mu_{\eta}}{12 f_{\pi}^{2}}\left\{64 M_{K}^{2}-2 M_{\pi}^{2}-27 u\right. \\
& \left.-\frac{81\left(s^{2}+t^{2}\right)-36(s+t) M_{\pi}^{2}+8 M_{\pi}^{4}}{12 M_{\eta}^{2}}+\frac{9\left(s^{2}+t^{2}\right)+24 u M_{K}^{2}-64 M_{K}^{4}}{2\left(M_{\pi}^{2}-M_{\eta}^{2}\right)}\right\}+\frac{4}{f_{\pi}^{4}}\left\{2 L_{2}^{r}\left(u-2 M_{K}^{2}\right)^{2}\right. \\
& \left.+\left(2 L_{1}^{r}+L_{2}^{r}+L_{3}\right)\left[\left(s-2 M_{K}^{2}\right)^{2}+\left(t-2 M_{K}^{2}\right)^{2}\right]-4 L_{4}^{r} u M_{K}^{2}-2 L_{5}^{r}\left(u-2 M_{K}^{2}\right) M_{\pi}^{2}-4\left[L_{5}^{r}-2\left(2 L_{6}^{r}+L_{8}^{r}\right)\right] M_{K}^{4}\right\} \\
& +\frac{186 s t-177 u^{2}+1032 u M_{K}^{2}-1648 M_{K}^{4}}{2304 f_{\pi}^{4} \pi^{2}}+\frac{1}{2 f_{\pi}^{4}}\left(u-2 M_{K}^{2}\right)^{2} \bar{J}_{K K}(u)+\frac{1}{288 f_{\pi}^{4}}\left\{60\left[s(2 s+t)+4 u M_{K}^{2}-8 M_{K}^{4}\right]\right. \\
& \times \bar{J}_{K K}(s)+2\left(9 s-8 M_{K}^{2}-M_{\pi}^{2}-3 M_{\eta}^{2}\right)^{2} \frac{\bar{J}_{\pi \eta}(s)}{3}+\left(9 s-2 M_{\pi}^{2}-6 M_{\eta}^{2}\right)^{2} \bar{J}_{\eta \eta}(s)+3\left[s\left(11 s+4 t-8 M_{K}^{2}\right)\right. \\
& \left.\left.-8\left(s+2 t-4 M_{K}^{2}\right) M_{\pi}^{2}\right] \bar{J}_{\pi \pi}(s)+[s \leftrightarrow t]\right\},
\end{aligned}
$$

and for $\bar{K}^{0} K^{0} \rightarrow K^{+} K^{-}$,

$$
\begin{aligned}
T_{\text {neu }}(s, t, u)= & \frac{2 M_{K}^{2}-u}{2 f_{\pi}^{2}}-\frac{\mu_{K}}{12 f_{\pi}^{2} M_{K}^{2}}\left\{5 s^{2}-s u+8 u^{2}-2 M_{K}^{2}(s+16 u)+36 M_{K}^{4}\right\}+\frac{\mu_{\pi}}{4 f_{\pi}^{2}}\left\{5\left(u-2 M_{K}^{2}\right)\right. \\
& \left.-\frac{11 s^{2}+4 t^{2}+4 s(2 t+u)-8(s+2 t) M_{K}^{2}}{12 M_{\pi}^{2}}+\frac{\left.24(s-2 t) M_{K}^{2}-9\left(s^{2}-2 t^{2}\right)+16 M_{K}^{4}\right\}}{8\left(M_{K}^{2}-M_{\pi}^{2}\right)}\right\} \frac{\mu_{\eta}}{12 f_{\pi}^{2}}\left\{9(s-u)+14 M_{K}^{2}\right. \\
& \left.-M_{\pi}^{2}-\frac{\left(9 s-2 M_{\pi}^{2}\right)^{2}}{12 M_{\eta}^{2}}+\frac{8 M_{K}^{2}\left(3 s-6 t+2 M_{K}^{2}\right)-9\left(s^{2}-2 t^{2}\right)}{2\left(M_{\pi}^{2}-M_{\eta}^{2}\right)}\right\}+\frac{2}{f_{\pi}^{4}}\left\{\left(4 L_{1}^{r}+L_{3}\right)\left(s-2 M_{K}^{2}\right)^{2}+2 L_{2}^{r}\left(u-2 M_{K}^{2}\right)^{2}\right. \\
& \left.+\left(2 L_{2}^{r}+L_{3}\right)\left(t-2 M_{K}^{2}\right)^{2}\right\}+\frac{4}{f_{\pi}^{4}}\left\{4 L_{4}^{r} s M_{K}^{2}-2 M_{K}^{4}\left[4 L_{4}^{r}+L_{5}^{r}-2\left(2 L_{6}^{r}+L_{8}^{r}\right)\right]-L_{5}^{r}\left(u-2 M_{K}^{2}\right) M_{\pi}^{2}\right\} \\
& -\frac{3\left(31 s^{2}+4 s u+16 u^{2}\right)-4 M_{K}^{2}\left(30 s+57 u-80 M_{K}^{2}\right)}{2304 f_{\pi}^{4} \pi^{2}}+\frac{\bar{J}_{K K}(s)}{6 f_{\pi}^{4}}\left[s(s-u)+4 M_{K}^{2}\left(2 M_{K}^{2}-t\right)\right]-\frac{\bar{J}_{\pi \eta}(s)}{432 f_{\pi}^{4}}
\end{aligned}
$$




$$
\begin{aligned}
& \times\left(9 s-8 M_{K}^{2}-M_{\pi}^{2}-3 M_{\eta}^{2}\right)^{2}+\frac{\bar{J}_{K K}(u)}{4 f_{\pi}^{4}}\left(u-2 M_{K}^{2}\right)^{2}+\frac{\bar{J}_{\pi \pi}(s)}{96 f_{\pi}^{4}}\left[s\left(7 s-4 t+8 M_{K}^{2}\right)+8\left(s+2 t-4 M_{K}^{2}\right) M_{\pi}^{2}\right] \\
& +\frac{\bar{J}_{\pi \pi}(t)}{24 f_{\pi}^{4}}\left(2 s+t-4 M_{K}^{2}\right)\left(t-4 M_{\pi}^{2}\right)+\frac{\bar{J}_{\pi \eta}(t)}{216 f_{\pi}^{4}}\left(9 t-8 M_{K}^{2}-M_{\pi}^{2}-3 M_{\eta}^{2}\right)^{2} \\
& +\frac{\bar{J}_{K K}(t)}{24 f_{\pi}^{4}}\left[t(s+2 t)+4 u M_{K}^{2}-8 M_{K}^{4}\right]+\frac{\bar{J}_{\eta \eta}(s)}{288 f_{\pi}^{4}}\left(9 s-2 M_{\pi}^{2}-6 M_{\eta}^{2}\right)^{2} .
\end{aligned}
$$

[1] S. Weinberg, Physica A 96, 327 (1979).

[2] J. Gasser and H. Leutwyler, Ann. Phys. (N.Y.) 158, 142 (1984).

[3] J. Gasser and H. Leutwyler, Nucl. Phys. B250, 465 (1985); B520, 517 (1985); B520, 539 (1985).

[4] H. Leutwyler, contribution to the Festschrift in honor of B. L. Ioffe, hep-ph/0008124; A. Dobado, A. Gómez-Nicola, A. L. Maroto, and J. R. Peláez, Effective Lagrangians for the Standard Model (Texts and Monographs in Physics) (SpringerVerlag, Berlin, 1997); A. Pich, Rep. Prog. Phys. 58, 563 (1995); U. G. Meißner, ibid. 56, 903 (1993).

[5] V. Bernard, N. Kaiser, and U. G. Meißner, Nucl. Phys. B364, 283 (1991); J. A. Oller and E. Oset, Phys. Rev. D 60, 074023 (1999); M. Jamin, J. A. Oller, and A. Pich, Nucl. Phys. B587, 331 (2000).

[6] J. A. Oller and E. Oset, Nucl. Phys. A620, 438 (1997); J. Nieves and E. Ruiz Arriola, Phys. Rev. D 63, 076001 (2001); Phys. Lett. B 455, 30 (1999); Nucl. Phys. A679, 57 (2000).

[7] T. N. Truong, Phys. Rev. Lett. 61, 2526 (1988); 67, 2260 (1991); A. Dobado, M. J. Herrero, and T. N. Truong, Phys. Lett. B 235, 134 (1990).

[8] A. Dobado and J. R. Peláez, Phys. Rev. D 47, 4883 (1993); 56, 3057 (1997).

[9] J. A. Oller, E. Oset, and J. R. Peláez, Phys. Rev. Lett. 80, 3452 (1998); Phys. Rev. D 59, 074001 (1999); 60, 099906(E) (1999)

[10] V. Bernard, N. Kaiser, and U. G. Meissner, Phys. Rev. D 43, 2757 (1991); Nucl. Phys. B357, 129 (1991); Phys. Rev. D 44, 3698 (1991).

[11] F. Guerrero and J. A. Oller, Nucl. Phys. B537, 459 (1999); B602, 641(E) (2001).

[12] M. Gell-Mann, Caltech Report No. CTSL-20, 1961; S. Okubo, Prog. Theor. Phys. 27, 949 (1962).

[13] H. Leutwyler, Phys. Lett. B 374, 163 (1996); Nucl. Phys. B (Proc. Suppl.) 64, 223 (1998); R. Kaiser, diploma work, Bern University, 1997.

[14] D. Iagolnitzer, J. Zinn-Justin, and J. B. Zuber, Nucl. Phys. B60, 233 (1973).

[15] A. M. Badalyan, L. P. Kok, M. I. Polikarpov, and Y. A. Simonov, Phys. Rep. 82, 31 (1982).

[16] Z. Xiao and H. Zheng, hep-ph/0103042; hep-ph/0107188.

[17] M. Boglione and M. R. Pennington, Z. Phys. C 75, 113 (1997); I. P. Cavalcante and J. Sa Borges, hep-ph/0101037.

[18] A. Dobado, A. Gómez-Nicola, and J. R. Peláez (in preparation).

[19] S. D. Protopopescu et al., Phys. Rev. D 7, 1279 (1973).
[20] P. Estabrooks and A. D. Martin, Nucl. Phys. B79, 301 (1974).

[21] A. D. Martin and E. N. Ozmutlu, Nucl. Phys. B158, 520 (1979).

[22] G. Grayer et al., in Experimental Meson Spectroscopy, edited by A. H. Rosenfeld and K. W. Lai, AIP Conf. Proc. No. 8 (AIP, New York, 1972), p. 5; presented at the 16th International Conference on High-Energy Physics, Batavia, Illinois, 1972, paper No. 768; B. Hyams et al., Nucl. Phys. B64, 134 (1973); W. Manner, presented at the 4th International Conference on Experimental Meson Spectroscopy, Boston, Massachusetts, 1974, CERN report; G. Grayer et al., Nucl. Phys. B75, 189 (1974).

[23] P. Estabrooks et al., in $\pi \pi$ Scattering, edited by D. K. Williams and V. Hagopian, AIP Conf. Proc. No. 13 (AIP, New York, 1973), p. 37.

[24] C. D. Frogatt and J. L. Petersen, Nucl. Phys. B129, 89 (1977).

[25] D. Cohen, Phys. Rev. D 22, 2595 (1980).

[26] A. Etkin et al., Phys. Rev. D 25, 1786 (1982).

[27] V. A. Polychronatos et al., Phys. Rev. D 19, 1317 (1979).

[28] W. Wetzel et al., Nucl. Phys. B115, 208 (1976).

[29] S. J. Lindebaum and R. S. Longacre, Phys. Lett. B 274, 492 (1992).

[30] D. Morgan and M. R. Pennington, Phys. Rev. D 48, 1185 (1993).

[31] W. Hoogland et al., Nucl. Phys. B126, 109 (1977).

[32] M. J. Losty et al., Nucl. Phys. B69, 185 (1974).

[33] L. Rosselet et al., Phys. Rev. D 15, 574 (1977).

[34] E865 Collaboration, P. Truöl et al., hep-ex/0012012; E865 Collaboration, S. Pisak et al., Phys. Rev. Lett. 87, 221801 (2001).

[35] R. Mercer et al., Nucl. Phys. B32, 381 (1971).

[36] P. Estabrooks et al., Nucl. Phys. B133, 490 (1978).

[37] H. H. Bingham et al., Nucl. Phys. B41, 1 (1972).

[38] S. L. Baker et al., Nucl. Phys. B99, 211 (1975).

[39] D. Aston et al., Nucl. Phys. B296, 493 (1988).

[40] D. Linglin et al., Nucl. Phys. B57, 64 (1973).

[41] T. A. Armstrong et al., Z. Phys. C 52, 389 (1991).

[42] G. Amorós, J. Bijnens, and P. Talavera, Nucl. Phys. B585, 293 (2000); B598, 665(E) (2001); B602, 87 (2001).

[43] J. Bijnens, G. Colangelo, and J. Gasser, Nucl. Phys. B427, 427 (1994).

[44] J. R. Peláez, J. A. Oller, and E. Oset, Nucl. Phys. A675, 92c (2000).

[45] Particle Data Group, D. Groom et al., Eur. Phys. J. C 15, 1 (2000).

[46] E791 Collaboration, R. E. Blanco et al., Phys. Rev. Lett. 86, 770 (2001); E791 Collaboration, C. Gobel, talk given at the IX 
International Conference on Hadron Spectroscopy, HADRON2001, Protvino, Russia, 2001, hep-ex/0012009.

[47] F. James, CERN Program Library Long Writeup D506, CERN, Geneva, Switzerland (1994).

[48] T. T. Wu and C. N. Yang, Phys. Rev. Lett. 13, 380 (1964).

[49] J. Gasser and U.-G. Meißner, Phys. Lett. B 258, 129 (1991).

[50] R. L. Jaffe, Phys. Rev. D 15, 267 (1977); 15, 281 (1977); E. van Beveren et al., Z. Phys. C 30, 615 (1986); S. Ishida et al., Prog. Theor. Phys. 98, 621 (1997).

[51] D. Black, A. H. Fariborz, F. Sannino, and J. Schechter, Phys.
Rev. D 58, 054012 (1998); M. Jamin, J. A. Oller, and A. Pich, Nucl. Phys. B587, 331 (2000); E. van Beveren and G. Rupp, hep-ex/0106077.

[52] S. N. Cherry and M. R. Pennington, Nucl. Phys. A688, 823 (2001).

[53] S. M. Flatté, Phys. Lett. 63B, 224 (1976).

[54] J. A. Oller, E. Oset, and J. R. Peláez, Phys. Rev. D 62, 114017 (2000).

[55] O. Dumbrajs et al., Nucl. Phys. B191, 301 (1981). 